\title{
Review
}

\section{The LATS1 and LATS2 tumor suppressors: beyond the Hippo pathway}

\author{
Noa Furth ${ }^{1}$ and Yael Aylon ${ }^{\star, 1}$
}

Proper cellular functionality and homeostasis are maintained by the convergent integration of various signaling cascades, which enable cells to respond to internal and external changes. The Dbf2-related kinases LATS1 and LATS2 (LATS) have emerged as central regulators of cell fate, by modulating the functions of numerous oncogenic or tumor suppressive effectors, including the canonical Hippo effectors YAP/TAZ, the Aurora mitotic kinase family, estrogen signaling and the tumor suppressive transcription factor p53. While the basic functions of the LATS kinase module are strongly conserved over evolution, the genomic duplication event leading to the emergence of two closely related kinases in higher organisms has increased the complexity of this signaling network. Here, we review the LATS1 and LATS2 intrinsic features as well as their reported cellular activities, emphasizing unique characteristics of each kinase. While differential activities between the two paralogous kinases have been reported, many converge to similar pathways and outcomes. Interestingly, the regulatory networks controlling the mRNA expression pattern of LATS1 and LATS2 differ strongly, and may contribute to the differences in protein binding partners of each kinase and in the subcellular locations in which each kinase exerts its functions.

Cell Death and Differentiation (2017) 24, 1488-1501; doi:10.1038/cdd.2017.99; published online 23 June 2017

Facts

- LATS1 and LATS2 proteins show extensive sequence similarity and share similar modes of post-translational modifications.

- The LATS genes are differentially regulated at the transcriptional level.

- LATS kinases engage divergent binding partners, although these effectors often converge on similar cellular processes.

- Whole-body deletion, as well as tissue-specific deletion, of either Lats1 or Lats2, reveals critical differences in the in vivo functions of the two kinases.

\section{Open Questions}

- Additional signaling pathways: what other functions do LATS kinases have beyond restricting YAP/TAZ activity?

- Redundant versus divergent function: what is the contribution of each kinase to distinct biological processes?

- Phosphorylation substrates of LATS: how is LATS kinase target recognition determined beyond simple amino-acid sequence motifs?

- Pro- versus anti-tumor effects: how does cellular context direct LATS toward apparently opposing functional outcomes?

In recent years, the LATS1 and LATS2 kinases have become the focus of intense research interest. They are gaining prominence due to their broad range of biological activities in cell cycle regulation, differentiation and motility, as well as the diverse pathological outcomes of their deregulation. LATS kinases are critical for organism fitness, genome integrity and cancer prevention. The core kinase module is evolutionarily conserved from yeast through flies to humans, although effectors and biological impact have expanded over the course of evolution.

The yeast ortholog of LATS, Dbf2 is localized to the spindle pole body (yeast centrosome) and regulates mitotic exit. ${ }^{1}$ Cdc15 (homolog of MST) is required for Dbf2 activation, ${ }^{2,3}$ and together they constitute a kinase module of the mitotic exit network. ${ }^{1,3}$ This module has been conserved in humans, manifested by LATS phosphorylation and activation by MST1/2 (MST) kinases. ${ }^{4}$ During evolution, this module recruited numerous different effectors, most notably the transcriptional coactivators YAP and TAZ, and extended its repertoire of biological functions. The Caenorhabditis elegans LATS, Ce-Wts-1, is associated with development, lifespan and body length control. ${ }^{5}$ Interestingly, nematodes lack YAP/TAZ, ${ }^{6}$ and Ce-Wts-1 exerts its function via effectors of the TGF-beta signaling pathway. ${ }^{5}$

Deletion of the Drosophila Warts (Wts, the fly ortholog of LATS) causes dramatic tissue overgrowth and abnormalities in cellular polarity. ${ }^{7,8}$ The fly Warts-Hippo (Hpo, MST ortholog) module exerts some of its functions via phosphorylation and inhibition of Yorkie (Yki, YAP and TAZ ortholog), ${ }^{9}$ while maintaining ancestral Yki-independent functions. ${ }^{10}$ The strong evolutionary conservation of the MST/LATS/YAP cascade (the Hippo pathway) is exemplified by the fact that human LATS 
proteins are able to rescue the loss of Wts functions in Drosophila. $^{11,12}$

As in other developmental pathways, complexity tends to increase over evolution. This is evidenced by the existence of additional components impacting the Hippo pathway, a diversity that might have been facilitated by duplication of the single ancestral LATS gene into two paralogs (coinciding with the duplication of other Hippo components, i.e., MST, TEAD and $Y A P$ ) during deuterostome evolution. ${ }^{6}$ Genetic studies in mice have underscored the functional differences between the duplicated LATS kinases. Loss of Lats2 is embryonic lethal on or before embryonic day E12.5, and this lethality is postulated to result from aberrant proliferation, mitotic defects and accumulated genomic instability. ${ }^{13,14}$ In contrast, Lats1-null mice are viable. However, they suffer from developmental defects such as infertility, growth retardation, pituitary dysfunction and lack of ductal structures in the mammary gland. In addition, Lats1-/- mice are prone to spontaneous and oncogene-induced sarcomas. ${ }^{15}$

In this review, we examine the features of LATS1 and LATS2, some of which are redundant (presumably representing a common primordial LATS function), and others distinct (presumably acquired in the course of evolutionary diversification). The common ability of both LATS kinases to repress YAP/TAZ has been studied extensively (reviewed recently in Zanconato et al. ${ }^{16}$ and Meng et al. ${ }^{17}$ ). Therefore we will focus mainly on LATS utilization of effectors other than YAP/TAZ, and the impact of those interactions on cell fate.

\section{Protein Structure and Post-Translational Modifications of LATS Kinases}

Human LATS1 and LATS2 are Ser/Thr kinases of the AGC subfamily, most closely related to the nuclear Dbf2-related kinases (NDR1/2). ${ }^{18}$ While LATS1 and LATS2 share extensive sequence similarity within their kinase domain ( $85 \%$ similarity) located at the $\mathrm{C}$ terminus of the proteins, the $\mathrm{N}$ terminus portion displays significantly lower conservation (Figure 1 and detailed in Table 1). ${ }^{19,20}$ Immediately carboxyterminal to the catalytic domain of both kinases is a hydrophobic motif; this pattern is akin to other AGC kinases such as AKT, S6K1 and PKC. ${ }^{18}$ Within the lowly conserved amino $(\mathrm{N})$ terminus, there are two stretches of conserved sequences (LCD1 and 2) that are required for proper LATS regulation and function. ${ }^{21,22}$ Also within the $\mathrm{N}$ terminus, both LATS1 and LATS2 harbor evolutionarily conserved ubiquitin-associated domains. Such domains are known to bind ubiquitinated proteins and may function in LATS activation. ${ }^{23}$ Interestingly, each of the kinases possesses unique features, which may facilitate different protein-protein interactions; the $\mathrm{N}$ terminus of LATS1 contains a proline-rich domain, ${ }^{24}$ while a unique PAPA repeat is found in LATS2. ${ }^{20}$ Furthermore, LATS2 encodes one, and LATS1 encodes two, PPxY motifs; these are essential for interaction with the WW hydrophobic pockets of YAP, TAZ and other Hippo pathway components. ${ }^{25}$

Superimposed on the amino-acid sequence is a combinatorial 'code' of post-translational modifications governing LATS activity (Figure 1; Table 1). Upstream signals such as cell cycle progression, cytoskeleton alterations and growth signals shape this code, defining different cellular outcomes.

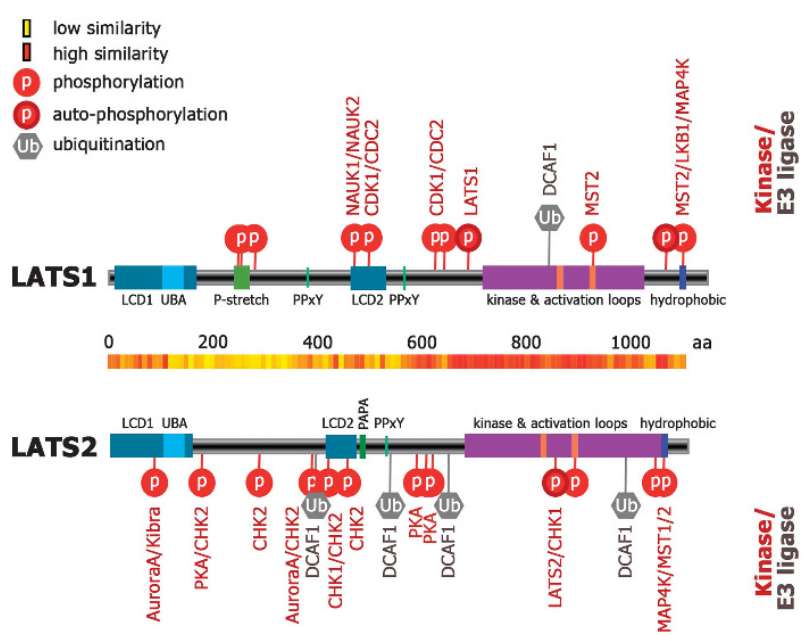

Figure 1 Schematic comparison of human LATS1 and LATS2 protein structures. Structural motifs, as defined by UniProtKB database, are represented as solid boxes on gray background. Reported phosphorylation sites are designated as red lollipops, with the phosphorylating kinase indicated above in dark red. Ubiquitination sites are gray denoted by hexagons, with the reported E3 ligase indicated above in dark gray. The heatmap between the LATS1 and LATS2 protein schemes represents the similarity of the aligned sequences, where dark orange represents high and yellow represents low amino-acid similarity. Similarity was calculated using the WatermanEggert local alignment application (EMBOSS explorer), comparing LATS1 (O95835-1) and LATS2 (Q9NRM7). Numbers above heatmap represent amino-acid position

LATS1 and LATS2 share dual phosphorylation-autophosphorylation mechanisms that are commonly employed by a subset of AGC kinases (including the aforementioned). ${ }^{26}$ MST-dependent phosphorylations of LATS (S909/T1079 on LATS1 and T1041 on LATS2) increase its kinase activity. ${ }^{4,27}$ Subsequently, MOB1 binding to the LATS hydrophobic domain relieves LATS autoinhibition and facilitates activating autophosphorylation (LATS1 residues S674 and S1049; LATS2 residue S835). ${ }^{28}$ In humans and flies, recruitment of LATS to the plasma membrane promotes MST-dependent phosphorylation and activation. ${ }^{29}$ PP2A-mediated dephosphorylation of these sites may counter MST-mediated phosphorylation to quench LATS1 activation. ${ }^{28,30}$ The effect of PP2A on LATS2 phosphorylation status has not been examined.

Importantly, MST are neither obligatory nor the sole LATS activators. For instance, deletion of Mst in mouse liver results in YAP hyperactivity without reduction in LATS phosphorylation status. ${ }^{31}$ In line with this, MAP4Ks phosphorylate both LATS1 and LATS2 hydrophobic motifs resulting in their activation and YAP inhibition. ${ }^{32}$ Similarly, phosphorylation of LATS2 by PKA bypasses MST to augment LATS2 kinase activity toward YAP. ${ }^{33}$ Other MST-independent phosphorylation of LATS may also result in cellular activities that are not related to YAP/TAZ regulation. For example, $\mathrm{CHK} 1 / 2$ phosphorylation of LATS2 S408 is associated with DNA damageinduced apoptosis. ${ }^{34}$ Likewise, LATS2 is activated by CHK1 and ATR in response to oncogenic H-RAS. ${ }^{35,36}$

Additional phosphorylation events of LATS1 and LATS2 relate to mitotic progression. A subset of molecules of both LATS1 and LATS2 is located at the centrosome, ${ }^{13,24}$ an organelle known for its crucial role in cell division. ${ }^{37}$ In mitosis, 


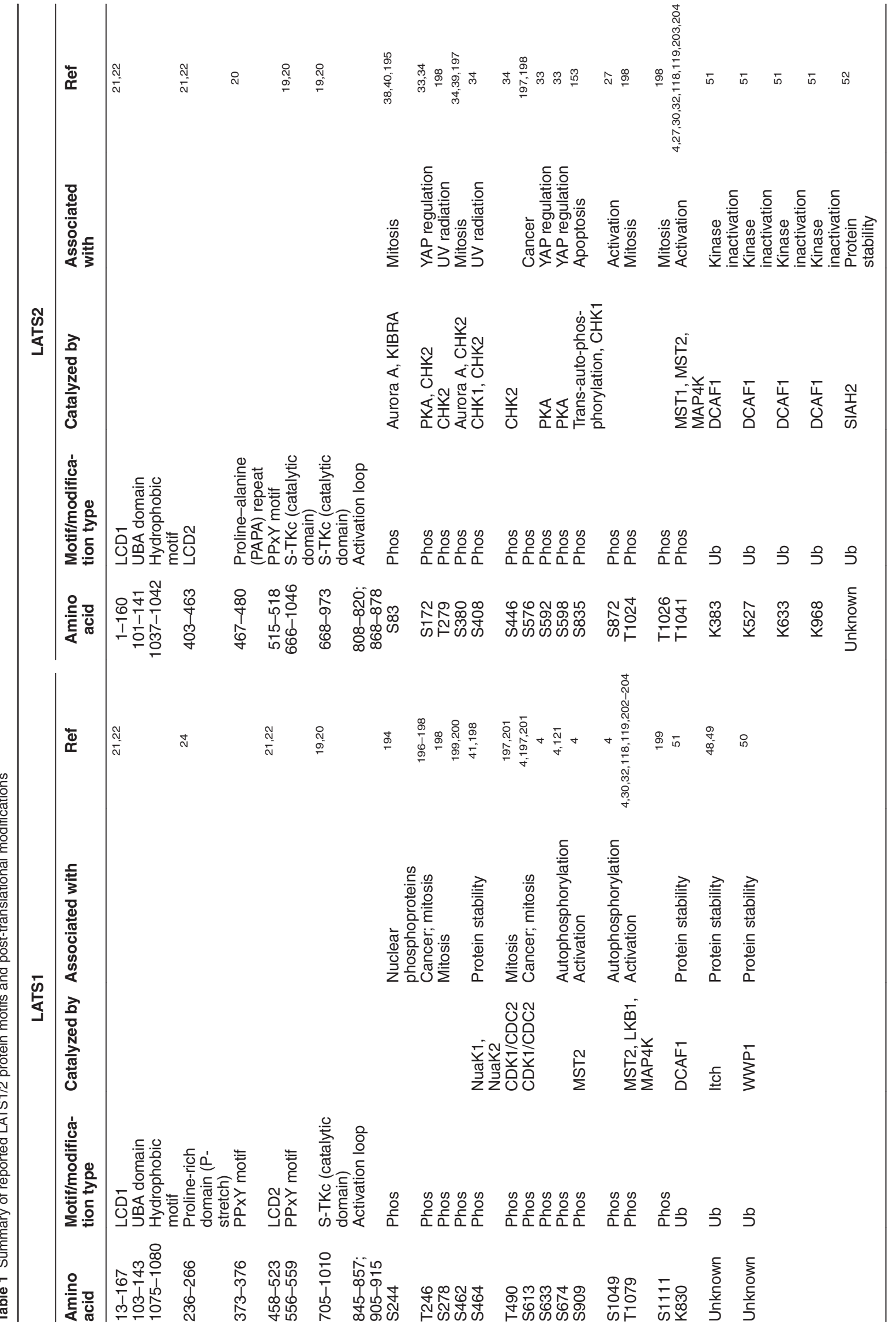


LATS1 (but not LATS2) is phosphorylated on T490 and S613 by $\mathrm{CDK} 1 / \mathrm{CDC} 2,{ }^{12}$ whereas LATS2 (but not LATS1) is phosphorylated on $\mathrm{S} 83$ and $\mathrm{S} 380$ by Aurora A kinase. ${ }^{38-40}$ This may reflect a general divergent and complementary phosphorylation pattern, whose functional consequences remain to be explored.

Differential phosphorylation of LATS1 and LATS2 also affects their stability. Thus, LATS1 phosphorylation on S464 by NUAK1 reduces its protein levels, ${ }^{41}$ whereas KIBRA stabilizes LATS2 by augmenting its phosphorylation and inhibiting its ubiquitination. ${ }^{42}$ Additionally, LATS protein stability and kinase activity can be bolstered by binding to heatshock proteins. For instance, both LATS kinases are clients of the molecular chaperone HSP90. ${ }^{43}$ Interestingly, MOB1 binding rescues LATS destabilization caused by HSP90 inhibition, ${ }^{43}$ suggesting that MOB1 also functions to stabilize the LATS proteins. On the other hand, destabilizers of LATS include the LIM domain-containing proteins Ajuba, Dachsous and Zyxin, ${ }^{44,45}$ which facilitate cell proliferation by reducing LATS protein levels and inhibition of LATS activity. ${ }^{45}$

LATS protein stability is regulated also through ubiquitination by a number of E3 ligases. Thus, NEDD4 ubiquitinates and promotes the degradation of both kinases, ${ }^{46,47}$ whereas additional E3 ligases with WW domains, such as ITCH and WWP1, specifically bind and destabilize LATS1. ${ }^{48-50}$ The WW-PPxY interaction between these E3 ligases and LATS1 might serve a dual purpose, by both decreasing LATS1 levels and displacing YAP/TAZ from its PPxY-binding site. Interestingly, CRL4-DCAF1 performs inhibitory ubiquitination of both kinases. ${ }^{51}$ However, whereas LATS1 is polyubiquitinated and directed to proteasomal degradation, LATS2 is oligoubiquitinated at multiple sites, resulting in kinase inactivation without enhanced degradation. This might reflect a cellular mechanism to free YAP/TAZ from LATS2 inhibition while retaining LATS2 kinase-independent functions. Yet, LATS2 is targeted for degradation by a distinct E3 ligase, SIAH2. ${ }^{52}$ Intriguingly, $\mathrm{SIAH} 2$ activity is associated with hypoxic response, ${ }^{53}$ and a decrease in LATS protein levels is critical for ROS-induced senescence. $^{54}$

\section{Regulation of LATS Gene Expression}

Classically, tumor suppressors may undergo loss of function due to genomic deletions or mutations, or through epigenetic silencing. Loss of heterozygosity of LATS1 was reported in ovarian, ${ }^{55,56}$ cervical $^{57}$ and breast ${ }^{58-60}$ cancer. Likewise, frequent copy number loss of LATS2 also occurs in breast, ${ }^{61}$ ovarian, ${ }^{62}$ hepatocellular ${ }^{63,64}$ and lung ${ }^{65}$ cancer, as well as in chronic lymphocytic leukemia. ${ }^{66}$

On the other hand, mutations in the LATS genes are relatively rare. However, due to the growing popularity of large genomic sequencing projects, evidence of LATS mutations in cancer is gradually emerging. ${ }^{67}$ In basal cell carcinoma of the skin, mutations occur specifically within the kinase domain of either LATS1 or LATS2 (16\% or $12 \%$, respectively), but rarely in both together. ${ }^{68}$ Interestingly, in other tumor types only one of the kinases is significantly mutated. This is exemplified in esophageal and non-small-cell lung cancer, where tumorspecific mutations were found in LATS2 but not LATS1. ${ }^{65,69}$ This further suggests that LATS1 and LATS2 may play distinct, non-redundant roles in some tumors. Nevertheless, the low rates of mutations in LATS genes emphasize that other mechanisms are dominant in reducing LATS activity, and it remains to be shown whether these mutations are driver rather than passenger mutations during tumorigenesis.

Promoter hypermethylation is another mechanism by which tumor suppressors are often inactivated. ${ }^{70}$ Such mode of inactivation has been documented for LATS1, ${ }^{71-73}$ LATS2, ${ }^{74,75}$ and in some cases for both, in various types of tumors. ${ }^{76-82}$ Importantly, downregulation of LATS expression has been associated with more aggressive cancer phenotypes. ${ }^{74,76-78,83}$ Promoter silencing can be mediated also by long non-coding RNAs (IncRNAs), which recruit the epigenetic machinery. Recently, it has been reported that the oncogenic IncRNAs PVT1, AGAP2-AS1 and LINC00673, whose elevated expression correlates with bad prognosis in non-small-cell lung and gastric cancers, tether polycomb repressive complexes to the LATS2, but not LATS1, promoter. ${ }^{84-86}$ Depletion of IncRNA reinstates LATS2 expression and causes p53-dependent cell death.

More broadly, LATS2 mRNA levels are exquisitely sensitive to tumor suppressive signaling, and are tightly regulated both transcriptionally and post-transcriptionally (Figure 2 and detailed in Table 2), while this seems to be less pertinent to LATS1 expression. Induction of LATS2 contributes to p53 tumor suppressive functions through a positive feedback mechanism, wherein the LATS2 protein promotes p53 stabilization by binding and inactivating the major p53 inhibitor MDM2, while p53 directly positively regulates the transcription of the LATS2 gene. ${ }^{87-89}$ In addition to regulating basal levels of LATS2, binding of p53 to the LATS2 promoter augments transcription in response to genotoxic, developmental and metabolic stresses. ${ }^{35,87,89-91}$

Like p53, FOXP3 also interacts directly with the LATS2 promoter to induce LATS2 expression. ${ }^{92}$ Interestingly, the levels of FOXP3 are positively regulated by $\mathrm{MST},{ }^{93}$ representing an additional mechanism by which MST promotes LATS2 activity. Intriguingly, also within the Hippo pathway, YAP/TAZ and their canonical partner transcription factor TEAD directly transactivate LATS2 (but not LATS1) gene expression. ${ }^{94,95}$ Hence, YAP/TAZ positively regulates the expression of one of their key negative regulators. Similarly, in fly wing disks, Wts expression is upregulated upon expression of activated Yki, and this depends on the fly ortholog of TEAD, Scalloped. ${ }^{94}$ It has been proposed that this negative feedback loop between LATS2 and YAP/TAZ serves to dampen the duration of YAP activity, $^{95}$ maintain homeostasis ${ }^{96}$ and render the Hippo pathway more robust, in order to resist the oncogenic effects of excessive YAP. $^{94}$

Nevertheless, positive regulation of LATS transcription does not always have a tumor suppressive outcome. For example, the LATS1 promoter can be transactivated by CUX1, a transcription factor associated with acceleration of S-phase and tumorigenesis. ${ }^{97,98}$ LATS2 overexpression in nasopharyngeal carcinomas was found to be associated with poor prognosis, ${ }^{99}$ and in metastatic human breast cancer cells high levels of LATS2 are associated with invasive and migratory capacities. ${ }^{100}$ Furthermore, according to publicly available TCGA data, LATS2 expression levels are elevated in glioblastoma, and the expression of both LATS1 and LATS2 


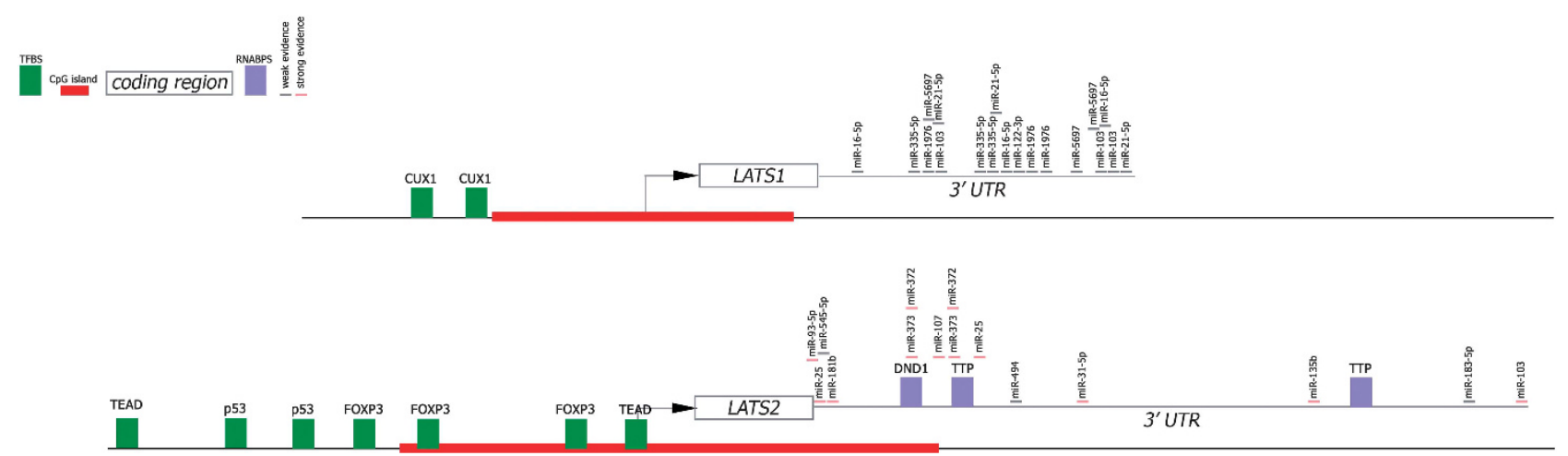

Figure 2 Scheme of human LATS1 and LATS2 genomic and mRNA structure. DNA is represented as a single black line. CpG islands (GC content $>50 \%$ ), as defined by UCSC Genome Browser (GRCh37/hg19), are indicated by thick red lines, the length of which corresponds to the relative length of the LATS1 and LATS2 CpG stretch. Transcription factor binding sites (TFBS) are represented schematically as dark green boxes, whereas RNA-binding protein sites (RNABPS) are mauve colored. The coding regions of LATS1 and LATS2 are not drawn to scale, but the $3^{\prime}$ UTR is drawn in the same scale as the CpG island designation. miR binding sites with strong experimental documentation are indicated by an orange underline, whereas putative miR binding sites, documented in broader screens with less conclusive direct evidence (miRTArBase ${ }^{193}$ ), are indicated by light gray lines

is significantly augmented in stomach cancer. ${ }^{101}$ This suggests that, contrary to the common assignment of LATS1 and LATS2 as tumor suppressors, retention of high LATS expression may actually sometimes be beneficial to the tumor, at least in some settings.

\section{Post-Transcriptional Regulation of LATS mRNA}

Several RNA-binding proteins have been shown to affect LATS2 mRNA stability. Both Piwi-like 2 (PiwiL2), a protein which usually mediates gene silencing, and Deadend 1 (DND1) stabilize the LATS2 transcript. ${ }^{102,103}$ DND1 binds to the $3^{\prime}$ UTR to protect LATS2 mRNA from microRNA (miR)mediated repression. ${ }^{104,105}$ On the other hand, TTP, an AU-rich domain RNA-binding protein, promotes the degradation of LATS2 mRNA by binding to its $3^{\prime} U T R{ }^{106}$ Interestingly, the DND1-binding site overlaps not only with miR target regions but also with one TTP binding site, consistent with the notion that multiple layers of RNA-binding proteins and miRs are in place to safeguard and modulate LATS2 mRNA levels.

Strong evidence exists that at least four miRs directly bind the LATS2 mRNA $3^{\prime}$ UTR to repress LATS2 expression (Figure 2 and detailed in Table 2). One miR, miR-135b, targets the mRNA of LATS2, ${ }^{107}$ as well as of additional components within the Hippo pathway (for instance MOB1 and NDR2 ${ }^{108}$ ), making it a 'Hippo-centric miR'. In contrast miR-31, an oncogenic miR overexpressed in numerous cancers, ${ }^{109}$ specifically targets LATS2 mRNA. ${ }^{110}$ Additionally, miR-372 and miR-373 have been shown to inhibit LATS2 mRNA, causing reduction of LATS2 expression and protein levels in testicular germ cell tumors, ${ }^{111}$ and in cell lines derived from gastric cancer ${ }^{112}$ and esophageal cancer. ${ }^{113}$

In contrast to LATS2, strong evidence of direct targeting of the LATS1 mRNA 3'UTR by miRs is lacking. This may, in part, be due to the difference in length of the $3^{\prime}$ UTRs (LATS1 814 nucleotides versus LATS2 1838 nucleotides, Figure 2), which might render the LATS2 mRNA more vulnerable to miRmediated inhibition. This supports the notion that, subsequent to the diversification of the ancestral LATS into two genes, evolution has shaped each of these genes to receive inputs from different signaling modules, thereby expanding substantially the connectivity of the Hippo pathway and providing it with a broader portfolio of 'networking' opportunities. Indeed, consistent with such conjecture, although both LATS1 and LATS2 3'UTRs are each highly conserved across different species, there is a very low similarity between them (only $3 \%$ similarity between the $3^{\prime}$ UTRs of human LATS1 and LATS2, according to the BLAST algorithm).

Interestingly, some miRs can have an indirect positive impact on LATS1 expression. For instance, miR-106b targets ITCH mRNA, encoding an E3 ligase that promotes LATS1 degradation, and in this way positively modulates LATS1 protein levels. ${ }^{114}$ Likewise, miR- 9 and miR-137 suppress the translation of $C U L 4 A$, a negative regulator of LATS $1 .{ }^{115}$ Notably, miR-9-3p (processed from the complementary strand of miR-9) targets TAZ mRNA; ${ }^{116}$ thus, both strands of miR-9 function to reinforce the tumor suppressive potential of the Hippo pathway and quench the output of its oncogenic effectors.

\section{LATS Protein Interactions and Cellular Localization}

Pathway analysis of fly Warts binding partners (data from Kwon et al. ${ }^{117}$ ) revealed an enrichment of metabolic and DNA repair pathways. Some of these functions may be conserved in mammals, as energy stress and DNA damage have been shown to activate LATS. ${ }^{118-121}$ Two studies have provided comprehensive pictures of the mammalian Hippo signaling interactome (refs 122,123 and Figure 3). As expected, proteins binding to both LATS1 and LATS2 are enriched for 'Hippo signaling'. However, proteins binding exclusively to LATS1 or LATS2 are quite different in their pathway enrichment. Thus, proteins associated with LATS1, but not LATS2, are related to Estrogen signaling, whereas LATS2 has Evolved a divergent interactome related to cell cycle, metabolism and p53. Of note, in both of these studies, the number of unique LATS2 interacting proteins was higher than of unique LATS1 interactors. Although this might have arisen from technical 

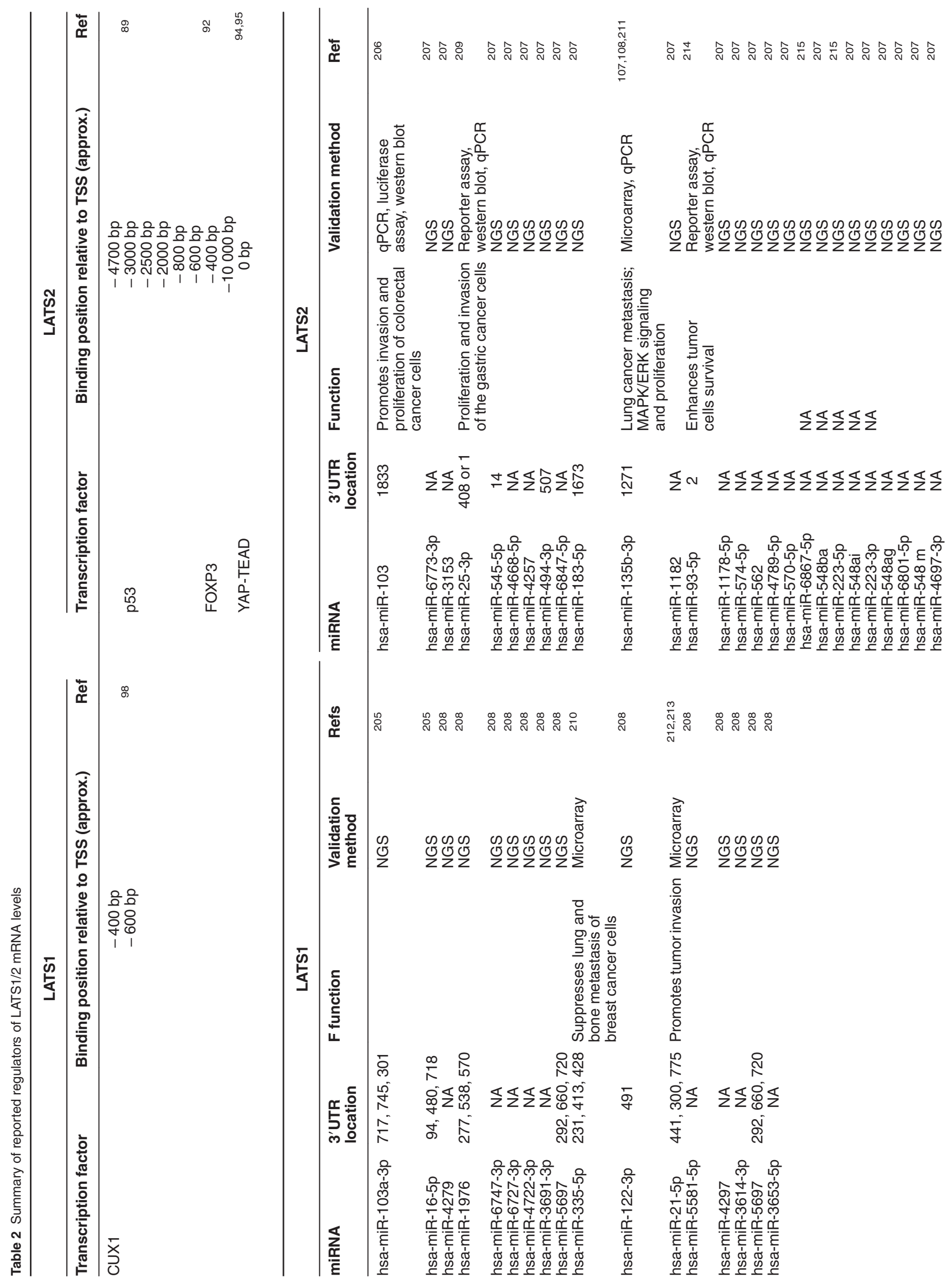
reasons, it also suggests inherent differences between the kinases. Hence, as is also the case for regulation by miRs, evolution following the gene duplication event may have resulted in a broader spectrum of LATS2-binding partners, in order to increase its networking capabilities.

Among other things, choice of binding partners both is affected by, and affects, protein subcellular localization. Both LATS kinases have been detected on centrosomes, ${ }^{13,24}$ which are presumably associated with their role in regulation of mitosis. Both can also be tethered to the plasma membrane ${ }^{29,124}$ or localize to the cytoplasm. ${ }^{21,125}$ It is commonly assumed that LATS kinases phosphorylate YAP/ TAZ in the cytoplasm. Yet, phosphorylation-dependent activation of LATS has been observed in the nucleus, ${ }^{51,126}$ while dephosphorylation of LATS1 and subsequent activation of YAP/TAZ can occur both in the nucleus and cytoplasm. ${ }^{127}$ Furthermore, LATS1 was recently shown to localize to either the nucleus or the cytoplasm of mammary epithelial cells, depending on cell lineage. ${ }^{128}$

Many of the functions unique to LATS2 have been attributed to its nuclear localization ${ }^{20}$ and its interaction with nuclear proteins. Upon mitotic or oncogenic stress, nuclear LATS2 potentiates the activity of the tumor suppressor p53..$^{35,36,89}$ In addition, nuclear LATS2 regulates chromatin dynamics by binding to polycomb repressive complex 2 (PRC2). ${ }^{129}$ Nuclear LATS2 was shown to restrict oncogenic $\beta$-catenin signaling by disrupting the chromatin-bound $\beta$-catenin-BCL9 complex. ${ }^{130}$ Accordingly, cardiac muscle-specific conditional knockout of Lats2 generates an elevated Wnt signature, ${ }^{131}$ and LATS2 expression is inversely correlated with the levels of Wnt target genes in human colorectal cancer. ${ }^{130}$ In contrast, in similar experiments LATS1 was not shown to bind chromatin or restrict $\beta$-catenin-induced transcription. ${ }^{130}$

Also within the nucleus, LATS2 restrains steroid rector transcriptional activity. In the prostate, LATS2 inhibits androgen receptor chromatin binding and transcriptional activity, ${ }^{132}$ while in breast tissue it modulates estrogen receptor (ER) activity. ${ }^{133}$ More recently, LATS kinases have been shown to restrict the activity of ER by binding and promoting its degradation. ${ }^{128}$ These studies implicate a nuclear function of LATS kinases in cell lineage commitment and in preventing the malignant progression of breast and prostate cancers. ${ }^{128,132}$

Together, a spectrum of subcellular localizations enables LATS kinases to impact a variety of physiological functions. ${ }^{134}$

\section{Cell cycle Regulation and Apoptosis}

Both LATS1 and LATS2 are involved in processes related to different stages of the cell cycle. Inhibition of CycE/CDK2 activity by LATS1 and LATS2 limits G1/S transition, under basal $^{21}$ as well as potentially genotoxic conditions. ${ }^{120,121}$ In addition, LATS2 phosphorylation of DYRK1A promotes the assembly of the DREAM complex, which represses the expression of S-phase E2F target genes to promote senescence. ${ }^{135}$

Multiple studies have linked LATS kinases to mitosis. Both LATS1 and LATS2 can bind to CDC25B ${ }^{136}$ and phosphorylate CDC26, ${ }^{137}$ master regulators of mitotic exit. Other studies suggest distinct modes of action for LATS1 and LATS2 during mitotic transition. ${ }^{138-140}$ In this scenario, LATS2 is 


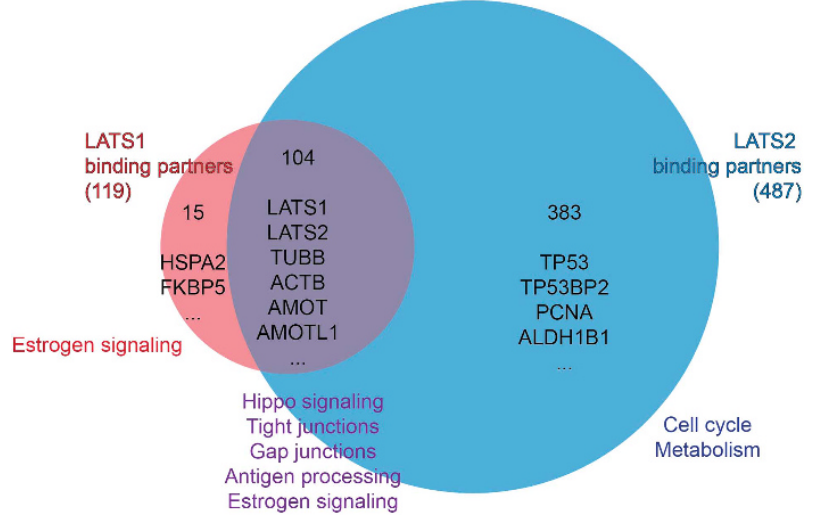

Figure 3 The protein interactome of LATS kinases. The Venn diagram depicts the overlap between putative LATS1 (119, light red circle) and LATS2 (487, light blue circle) binding partners in mammalian cells, as reported by Couzens et al. $^{123}$ and Wang et al. ${ }^{122}$ The numbers of common or exclusive binding partners and specific notable examples are indicated. Key-enriched biological processes (KEGG pathway database) are shown below the diagram

phosphorylated by Aurora A, and phospho-LATS2 translocates to the spindle along with LATS1, which phosphorylates Aurora $B$ to ensure proper cytokinesis. ${ }^{39}$ The above-described mitotic function of LATS kinases in mammalian cells is reminiscent of the role of yeast $\mathrm{Dbf} 2,{ }^{3}$ and may therefore represent an ancient dedication of the pathway to governing mitotic exit, which has been preserved in all metazoans. ${ }^{6}$

Since one of the functions of the mitotic exit pathway is to ensure that cytokinesis does not occur before proper partitioning of the genetic material, it may not be surprising that LATS1 and LATS2 are crucial in sensing mitotic stress that occurs in response to microtubule poisons such as nocodazole or during hyperproliferation owing to oncogene activation. ${ }^{35,89,141}$ These functions are strongly associated with the ability of LATS2 to promote activation of p53dependent checkpoints, which may lead to either G1/S arrest or apoptosis. ${ }^{35,89}$ Indeed, extra chromosomes resulting from cytokinesis failure are sufficient to activate the Hippo pathway via the LATS2-p53 axis. ${ }^{142}$ Together with its ability to be transactivated by $\mathrm{p53}$, this constitutes a LATS2-p53 tumorsuppressive positive feedback loop. In line with this, nuclear LATS2 can associate with p53 on the p21 promoter to inhibit proliferation under stress conditions. ${ }^{143}$ In this context, it is interesting to note that overexpression of kinase-dead LATS1 suppresses the ability of cells to induce p53 in response to mitotic stress. ${ }^{144}$ Although this suggests that p53 is also sensitive to LATS1, it remains plausible that the effects of kinase-dead LATS1 might be due to dominant-negative inhibition of endogenous LATS2.

Alleviation of the MDM2-dependent inhibition of p53 can eliminate potentially transformed cells from the replicative pool. ${ }^{145}$ Sustained K-RAS signaling promotes LATS1/MDM2/ p53-dependent apoptosis. ${ }^{146}$ Likewise, expression of oncogenic H-RAS facilitates LATS2-dependent phosphorylation of the pro-apoptotic protein ASPP1, and drives p53-dependent apoptosis. ${ }^{36}$ Furthermore, ASPP1 can bind and inhibit LATS1mediated phosphorylation of YAP, resulting in increased YAP activity. ${ }^{147}$ This molecular wiring might exemplify another mechanism by which LATS2 indirectly modulates LATS1 activity.

Additional means by which LATS1 can impact apoptosis have been suggested. LATS1 is activated by death receptors downstream of RASSF1A and MST2. ${ }^{148}$ In turn, LATS1 increases the expression of the pro-apoptotic protein BAX. ${ }^{140}$ Furthermore, LATS1 binds and enhances the protease activity of Omi/HtrA2, ${ }^{149}$ a mitochondrial protein that is released into the cytoplasm during apoptosis. ${ }^{150}$ LATS1 also feedbacks to inhibit MST2 pro-apoptotic activities by phosphorylating RAF1 on Ser259. ${ }^{151}$ This phosphorylation promotes the inhibitory binding of RAF1 to MST2 and restricts RAF1 binding and activation of MEK signaling. Thus, by phosphorylating RAF1, LATS1 restricts both ERK-dependent cellular proliferation and MST2-dependent apoptosis. ${ }^{151}$

LATS2 can downregulate the expression of the antiapoptotic proteins BCL-xL and BCL2 by a mechanism that requires its kinase activity. ${ }^{152}$ Interestingly, the LATS2-p53 functional axis can regulate apoptosis not only through the downstream activation of p53 transcriptional target genes, but also by non-transcriptional mechanisms. In particular, following UV irradiation, LATS2 phosphorylates the p21 protein, encoded by a major p53 transcriptional target gene, to induce its degradation. ${ }^{153}$ In this way, cells bypass cell cycle arrest and are directed to die. Of note, the p53 family member p73 can act as potent inducer of apoptosis when bound to YAP. ${ }^{154,155}$ Interestingly, in leukemic cells, LATS2 promotes the pro-apoptotic activity of the p73-YAP complex. ${ }^{156}$

Surprisingly, inhibition of the p73-YAP complex by the LATS kinases can also have an anti-apoptotic affect. ${ }^{157}$ LATS2 can also inhibit DNA damage-induced apoptosis through inhibitory phosphorylation of $\mathrm{c}$-Abl. ${ }^{158}$ The tyrosine kinase $\mathrm{c}-\mathrm{Abl}$ is a strong inducer of the YAP-p73 pro-apoptotic axis in response to DNA damage. ${ }^{159,160}$ Specifically, phosphorylation of YAP Tyr357 by c-Abl potentiates the binding to p73 and induction of pro-apoptotic genes. ${ }^{159}$ Since, c-Abl and YAP can contribute or inhibit apoptosis, ${ }^{159,161-163}$ their inhibition by LATS kinases results in opposing outcomes. Overall, this highlights an interplay between LATS, YAP, p73 and c-Abl, whose eventual impact on apoptosis is highly cell context-dependent.

In sum, LATS kinases govern cell fate by manipulating both cell cycle and apoptosis. This becomes particularly important when cells are faced with replicative or oncogenic stress and must be removed from the proliferative pool in a cost-effective manner and with the least harm to the organism as a whole.

\section{Migration and EMT}

Epithelial to mesenchymal transition (EMT) and migration, two important features in development and oncogenic transformation, are both regulated by LATS kinases. Mechanistically, human LATS1 and Drosophila Warts can bind to actin and inhibit actin polymerization. ${ }^{164,165}$ In mammals, reduced LATS expression promotes cell migration by altering the functional state of $p 53^{101}$ and by increasing the activity of the YAP/TAZ transcriptional module. ${ }^{166}$ It is noteworthy that YAP/TAZ sensitivity to cytoskeleton and cell motility dynamics is critical to their role in mechanosensing, some of which is LATSindependent. ${ }^{167}$ Overall, the inhibitory effects of LATS kinases 
on cell migration are in line with their assignment as tumor suppressors.

Surprisingly, LATS2 can also potentiate the activity of tumorpromoting factors and augment EMT. In fact, LATS2 was reported to increase the cell invasive capacities of metastatic breast cancer cell lines harboring mutant $p 53 .{ }^{100}$ In that case, the underlying mechanism was proposed to be the phosphorylation of SNAIL1 by LATS2, leading to increased SNAIL1 stability, nuclear localization and transcriptional activity. ${ }^{100}$

\section{Embryogenesis and Stem Cells}

The LATS-YAP/TAZ axis plays a key role in patterning of mammalian embryos and determining cell lineage and differentiation, as exemplified in mouse studies. ${ }^{168-170}$ In support of this, inhibition of Lats 1 and Lats2 expression in early embryos results in irreversible lineage misspecification and aberrant polarization of the inner cell mass. ${ }^{170}$ Specifically, LATS2 seems to play a critical role in early embryogenesis. The pluripotent transcription factors OCT4 and NANOG bind a region near the Lats2 (but not Lats1) gene, and repress Lats2 expression. ${ }^{171}$ Accordingly, deletion of Lats2 (but not Lats 1) is embryonic lethal. ${ }^{13}$ Mouse embryonic stem cells (mESCs) lacking Lats2 display an altered chromatin landscape that retains $\mathrm{H} 3 \mathrm{~K} 4 \mathrm{me} / \mathrm{H} 3 \mathrm{~K} 27 \mathrm{me} 3$ bivalent histone marks; ${ }^{91}$ this may be related to the ability of LATS2 to associate with PRC2 to promote H3K27 tri-methylation. ${ }^{129}$ In line with this, mESCs lacking Lats2 are deficient in both sustaining pluripotency and responding to differentiation signals, ${ }^{91}$ suggesting a cellular mechanism for the embryonic lethality phenotype of Lats2 - /- mice. Importantly, inhibition of Yap/Taz activity fails to rescue the transcriptional defect of Lats2-/- mESCs; rather, the ability of LATS2 to maintain mESC homeostasis is mediated by the LATS2-p53 functional axis. $^{91}$

Members of the miR-290 family of microRNAs (mouse orthologues of human miR-372/373) are highly expressed in undifferentiated mESCs, and can promote their proliferation by potentiating $\mathrm{G} 1$ to $\mathrm{S}$ transition. Downregulation of Lats 2 by these miRs contributes to pluripotency by interfering with the ability of LATS2 to promote G1 arrest. ${ }^{172}$ Intriguingly, on the other hand, reprogramming to induced pluripotent stem cells has been shown to be inhibited by LATS2 via a p53-independent mechanism that does not accelerate cell proliferation. ${ }^{173}$

In more advanced stages of development, such as lineagespecific differentiation, LATS2 was shown to contribute to the differentiation process. For example, LATS2 inhibits preadipocyte proliferation and promotes adipocyte differentiation by inducing a PPARy pro-adipogenic transcriptional program. ${ }^{174,175}$ Although this was shown to be mediated by cytoplasmic retention of TAZ, it still remains to be investigated whether this function is shared also with LATS1. Altogether, LATS2 plays a unique role in embryonic stem cells and in differentiation.

\section{Tissue-Specific Roles of LATS Kinases}

YAP/TAZ are key regulators of liver size and, when hyperactivated, can drive liver tumorigenesis. ${ }^{163,176}$ Thus, it is not surprising that inactivation of both LATS kinases in liver cells leads to failure of proper differentiation and augments proliferation. ${ }^{177,178}$ Embryonic deletion of both kinases in the mouse liver results in neonate lethality. ${ }^{177}$ In adult livers, acute deletion of Lats 1/2 results in dedifferentiation of hepatocytes into immature biliary epithelial cells, fibrosis and lethal liver impairment. ${ }^{178}$ LATS2 also has additional hepatic functions, which are not mediated by YAP/TAZ activity and are not shared with LATS1. For example LATS2, but not LATS1, inhibits hepatic cholesterol accumulation by binding and quenching the transcriptional activity of SREBP1 and SREBP2, transcription factors that are master regulators of lipid and cholesterol homeostasis. ${ }^{90}$ Consequently, mice lacking Lats2 in the liver have deregulated cholesterol metabolism and are prone to fatty liver disease, suggesting that LATS2 plays a role in metabolic homeostasis. ${ }^{85}$

As in the liver, Lats1 and Lats2 are essential also in the kidney ureteric bud lineage: deletion of both Lats genes results in severe defects in branching morphogenesis, deregulated cell polarity and hyperactivation of YAP and TAZ. ${ }^{179}$

In the heart, inactivation of both LATS kinases reflects a role for LATS in restricting cardiomyocyte renewal and regeneration. ${ }^{180}$ Interestingly, the individual functions of each kinase in cardiomyocytes may not be fully redundant, since inactivation of Lats2 is sufficient to cause myocardial expansion ${ }^{131}$ and Lats2 overexpression negatively regulates ventricular mass in the heart. ${ }^{181}$ Furthermore, the kinase activity of LATS2 is required for YAP's ability to regulate coronary vascular formation. ${ }^{182}$ In line with these observations, expression of Lats2, but not Lats1, promotes apoptosis in cultured cardiomyocytes. ${ }^{181}$

Both LATS kinases are expressed ubiquitously throughout different human tissues, ${ }^{134}$ except the spleen in which neither kinase is detected (Human Protein Atlas available at: www. proteinatlas.org). ${ }^{183}$ Protein levels differ, with very few tissues showing similar trends of expression between LATS1 and LATS2. While LATS1 protein is detected in high levels throughout most tissues, LATS2 protein levels seem to vary, with highest expression in the gastrointestinal tract and the brain. ${ }^{183}$ The functional impact of each kinase in different tissues remains to be further examined.

\section{Conclusion}

The great interest in the Hippo pathway components has generated a wealth of new information. Yet, many of the studies have focused exclusively on the pathway downstream effectors YAP and TAZ, and LATS kinases function, if addressed at all, has been examined merely in the light of their effect on YAP/TAZ. Furthermore, most studies employ only one LATS gene or protein, making it difficult to identify true differences between LATS1 and LATS2. In this review, we have tried to tease out and analyze discrete characteristics of LATS1 and LATS2, as recorded in the literature to date. We show that although, as expected, there does exist substantial functional overlap between these two paralogs, many of their features are nevertheless distinct.

The LATS duplication event set the stage for evolution to 'teach' us about LATS function. Gene duplication establishes a platform for exploring genetic novelty, while augmenting 
genomic robustness by buffering paralogs. ${ }^{184}$ Actually, evolution pushes the duplicated genes toward diversification, as total redundancy among duplicates is both genetically unfavorable and potentially disruptive to biochemical pathways due to dosage sensitivity. ${ }^{185}$ Together, this suggests that the second copy is liberated from selective pressure and can evolve novel functions, as long as any ensuing functional losses can be complemented by the other copy. ${ }^{186}$ Interestingly, alterations in gene expression often precede functional changes in paralog evolution. ${ }^{187}$

LATS1 and LATS2 embody this evolutionary format. The most striking differences between LATS1 and LATS2 occur on the transcript level. The difference in transcription factors regulating LATS1 versus LATS2 may represent the necessity to keep tight reigns on the 'brakes' and 'gas' of proliferation signals by maintaining proficient levels of LATS in both conditions. Further indication of tight regulation on the transcriptional level is evident in their 3'UTRs: LATS2 contains a long, highly regulated 3'UTR, whereas the shorter LATS1 UTR may evade, at least to some extent, negative (miR) or positive (RNA-binding proteins) regulation. Interestingly, lengthening of 3'UTRs has been associated with increased morphological complexity over evolution ${ }^{188}$ and might be linked to observations that regulatory motifs in UTRs are often conserved in genes within similar functional pathways. ${ }^{189}$ It will be interesting to examine the possibility that LATS2 has evolved functions that enable it to be co-regulated within the context of a larger functional gene family; this concept is illustrated by the observation that miR-372/3 commonly targets LATS2 as well as other factors that are critical in stem cell differentiation. ${ }^{190}$

The divergent expression patterns of LATS1 and LATS2 might contribute to their likelihood of encountering distinct binding partners that, in turn, might tether the two LATS proteins to different cellular localizations and facilitate their distinct functions. This is illustrated by the specialized connection of LATS1 to estrogen signaling, and of LATS2 to stem cell differentiation and to the p53 network. It is important to note, however, that even in these 'dedicated' interactions, there is substantial redundancy between LATS1 and LATS2, which probably underpins their ability to serve as partial backups for each other. Thus, LATS2-specific interacting partners are not enriched in estrogen signaling, ${ }^{122,123}$ yet both LATS1 and LATS2 have been shown to regulate the stability of the ER. ${ }^{128}$ Likewise, p53 exclusively binds LATS2 but not LATS $1^{122}$ and transcriptionally activates the LATS2 but not LATS1 promoter, $^{89}$ but LATS1 can nevertheless modulate p53-dependent apoptosis. ${ }^{146}$ Similarly, OCT4 and NANOG repress Lats2 but not Lats 1 expression, ${ }^{171}$ which is essential for proper embryonic development, but Lats 1 is also important for embryogenesis, since re-expression can rescue Lats depletion phenotype in early embryogenesis. ${ }^{170}$

In fact, a considerable proportion of LATS functions intersect on different elements of the same pathway. For instance, LATS2 is phosphorylated by Aurora A and LATS1 phosphorylates Aurora B. ${ }^{39}$ Both Aurora kinases impact mitotic progression, however Aurora A associates with the spindle poles to regulate entry into mitosis and spindle assembly, whereas Aurora B regulates chromosome cohesion and cytokinesis. $^{191}$ Therefore, although the LATS1/2-specific mechanisms may have diverged, in most cases the broader physiological 'agenda' of the LATS kinases has been retained. Probably for these reasons, both LATS genes undergo selective silencing in cancer.

The LATS kinases restrict the 'canonical' Hippo effectors YAP and TAZ, and also control 'non-canonical' novel signaling pathways to integrate critical cellular processes. However, the distinction between 'canonical' and emergent LATS functions quickly becomes blurry. Some novel activities of LATS indirectly impinge on YAP/TAZ functions. ${ }^{36,90,147}$ Additionally, due to YAP-LATS2 feedback, hyperactivation of YAP is expected to also inherently affect LATS2 non-canonical functions. Furthermore, LATS2 has been shown to act upstream to LATS1 and enhance its kinase activity toward non-canonical effectors. ${ }^{39}$ Many non-canonical LATS kinaseregulated events are not associated with the $H X R X X S / T$ consensus LATS phosphorylation motif, ${ }^{192}$ suggesting that in these cases LATS substrate selection is shaped by factors other than just amino-acid sequence. Thus, complicated and multi-directional mechanisms are in place, even within the Hippo module itself.

Consequently, LATS-dependent cell fate decisions are the sum total of innumerous signaling inputs and outputs, the weight of each signal being determined (among many other factors) by cell density, cell type, developmental stage, neighboring cells and whether the cells are normal or transformed. Together, these complex signals lead to a vast and sometimes contradictory spectrum of LATS functions and activities. Some of the most striking examples are illustrated by the ability of LATS1 and LATS2 to both promote and inhibit apoptosis, ${ }^{139,152,153,157,158}$ and the ability of LATS2 to both augment and inhibit differentiation ${ }^{91,129}$ or cellular migration. ${ }^{100,101}$

Many open questions still remain to be answered. More meticulous studies need to be performed to accurately define LATS1 and LATS2 shared versus distinct functions. Advances in understanding LATS signaling may aid to resolve basic scientific enigmas such as how kinases choose phosphorylation substrates, how signaling pathways balance cell division, differentiation and proliferation, and how these pathways are skewed during cancerous transformation. Moreover, deciphering the details of LATS-mediated tumor suppression will hopefully elucidate opportunities for improved early detection, prognostication and treatment of cancer.

\section{Conflict of Interest}

The authors declare no conflict of interest.

1. Visintin R, Amon A. Regulation of the mitotic exit protein kinases Cdc15 and Dbf2. Mol Biol Cell 2001; 12: 2961-2974.

2. Rock JM, Lim D, Stach L, Ogrodowicz RW, Keck JM, Jones MH et al. Activation of the yeast Hippo pathway by phosphorylation-dependent assembly of signaling complexes. Science 2013; 340: 871-875.

3. Bardin AJ, Amon A. Men and sin: what's the difference? Nat Rev Mol Cell Biol 2001; 2 : 815-826.

4. Chan EHY, Nousiainen M, Chalamalasetty RB, Schäfer A, Nigg EA, Silljé HHW. The Ste20-like kinase Mst2 activates the human large tumor suppressor kinase Lats1. Oncogene 2005; 24: 2076-2086

5. Cai Q, Wang W, Gao Y, Yang Y, Zhu Z, Fan Q. Ce-wts-1 plays important roles in Caenorhabditis elegans development. FEBS Lett 2009; 583: 3158-3164.

6. Hilman D, Gat U. The evolutionary history of YAP and the Hippo/YAP pathway. Mol Biol Evol 2011; 28: 2403-2417. 
7. Bryant PJ, Watson KL, Justice RW, Woods DF. Tumor suppressor genes encoding proteins required for cell interactions and signal transduction in Drosophila. Development 1993: 239-249.

8. Justice RW, Zilian O, Woods DF, Noll M, Bryant PJ. The Drosophila tumor suppressor gene warts encodes a homolog of human myotonic dystrophy kinase and is required for the control of cell shape and proliferation. Genes Dev 1995; 9: 534-546.

9. Zhao B, Li L, Lei Q, Guan K-L. The Hippo-YAP pathway in organ size control and tumorigenesis: an updated version. Genes Dev 2010; 24: 862-874.

10. Dewey EB, Sanchez D, Johnston CA. Warts phosphorylates mud to promote pins-mediated mitotic spindle orientation in Drosophila, independent of Yorkie. Curr Biol 2015; 25: 2751-2762.

11. Staley BK, Irvine KD. Hippo signaling in Drosophila: recent advances and insights. Dev Dyn 2012; 241: 3-15.

12. Tao W, Zhang S, Turenchalk GS, Stewart RA St, John MA, Chen W et al. Human homologue of the Drosophila melanogaster lats tumour suppressor modulates CDC2 activity. Nat Genet 1999; 21: 177-181.

13. McPherson JP, Tamblyn L, Elia A, Migon E, Shehabeldin A, Matysiak-Zablocki E et al. Lats $2 / \mathrm{Kpm}$ is required for embryonic development, proliferation control and genomic integrity. EMBO J 2004; 23: 3677-3688.

14. Yabuta N, Okada N, Ito A, Hosomi T, Nishihara S, Sasayama $Y$ et al. Lats2 is an essential mitotic regulator required for the coordination of cell division. J Biol Chem 2007; 282 19259-19271.

15. St John MA, Tao W, Fei X, Fukumoto R, Carcangiu ML, Brownstein DG et al. Mice deficient of Lats1 develop soft-tissue sarcomas, ovarian tumours and pituitary dysfunction. Nat Genet 1999; 21: 182-186.

16. Zanconato F, Cordenonsi M, Piccolo S. YAP/TAZ at the roots of cancer. Cancer Cell 2016; 29: 783-803.

17. Meng Z, Moroishi T, Guan K-L. Mechanisms of Hippo pathway regulation. Genes Dev 2016; 30: 1-17.

18. Avruch J, Zhou D, Fitamant J, Bardeesy N, Mou F, Barrufet LR. Protein kinases of the Hippo pathway: regulation and substrates. Semin Cell Dev Biol 2012; 23: 770-784.

19. Hori T, Takaori-Kondo A, Kamikubo Y, Uchiyama T. Molecular cloning of a novel human protein kinase, $\mathrm{kpm}$, that is homologous to warts/lats, a Drosophila tumor suppressor. Oncogene 2000; 19: 3101-3109.

20. Yabuta N, Fujii T, Copeland NG, Gilbert DJ, Jenkins NA, Nishiguchi $\mathrm{H}$ et al. Structure, expression, and chromosome mapping of LATS2, a mammalian homologue of the Drosophila tumor suppressor gene lats/warts. Genomics 2000; 63: 263-270.

21. Li Y, Pei J, Xia H, Ke H, Wang H, Tao W. Lats2, a putative tumor suppressor, inhibits G1/S transition. Oncogene 2003; 22: 4398-4405.

22. Yabuta N, Mukai S, Okamoto A, Okuzaki D, Suzuki H, Torigata K et al. N-terminal truncation of Lats1 causes abnormal cell growth control and chromosomal instability. J Cell Sci 2013 126: 508-520.

23. Kim M, Kim M, Park S-J, Lee C, Lim D-S. Role of Angiomotin-like 2 mono-ubiquitination on YAP inhibition. EMBO Rep 2016; 17: 64-78.

24. Nishiyama Y, Hirota T, Morisaki T, Hara T, Marumoto T, lida S et al. A human homolog of Drosophila warts tumor suppressor, h-warts, localized to mitotic apparatus and specifically phosphorylated during mitosis. FEBS Lett 1999; 459: 159-165.

25. Sudol M. Newcomers to the WW domain-mediated network of the Hippo tumor suppressor pathway. Genes Cancer 2010; 1: 1115-1118.

26. Pearce LR, Komander D, Alessi DR. The nuts and bolts of AGC protein kinases. Nat Rev Mol Cell Biol 2010; 11: 9-22.

27. Hoa L, Kulaberoglu Y, Gundogdu R, Cook D, Mavis M, Gomez M et al. The characterisation of LATS2 kinase regulation in Hippo-YAP signalling. Cell Signal 2016; 28: 488-497.

28. Hergovich A, Schmitz D, Hemmings BA. The human tumour suppressor LATS1 is activated by human MOB1 at the membrane. Biochem Biophys Res Commun 2006; 345: 50-58.

29. Yin F, Yu J, Zheng Y, Chen Q, Zhang N, Pan D. Spatial organization of Hippo signaling at the plasma membrane mediated by the tumor suppressor Merlin/NF2. Cell2013; 154: 1342-1355.

30. Praskova M, Xia F, Avruch J. MOBKL1A/MOBKL1B phosphorylation by MST1 and MST2 inhibits cell proliferation. Curr Biol 2008; 18: 311-321.

31. Zhou D, Conrad C, Xia F, Park J-S, Payer B, Yin Y et al. Mst1 and Mst2 maintain hepatocyte quiescence and suppress hepatocellular carcinoma development through inactivation of the Yap1 oncogene. Cancer Cell 2009; 16: 425-438.

32. Meng Z, Moroishi T, Mottier-Pavie V, Plouffe SW, Hansen CG, Hong AW et al. MAP4K family kinases act in parallel to MST1/2 to activate LATS1/2 in the Hippo pathway. Nat Commun 2015; 6: 8357.

33. Kim M, Kim M, Lee S, Kuninaka S, Saya H, Lee $\mathrm{H}$ et al. cAMP/PKA signalling reinforces the LATS-YAP pathway to fully suppress YAP in response to actin cytoskeletal changes. EMBO J 2013; 32: 1543-1555.

34. Okada N, Yabuta N, Suzuki H, Aylon Y, Oren M, Nojima H. A novel Chk1/2-Lats2-14-3-3 signaling pathway regulates P-body formation in response to UV damage. J Cell Sci 2011; 124: $57-67$.

35. Aylon $\mathrm{Y}$, Yabuta N, Besserglick $\mathrm{H}$, Buganim $\mathrm{Y}$, Rotter $\mathrm{V}$, Nojima $\mathrm{H}$ et al. Silencing of the Lats2 tumor suppressor overrides a p53-dependent oncogenic stress checkpoint and enables mutant H-Ras-driven cell transformation. Oncogene 2009; 28: 4469-4479.

36. Aylon Y, Ofir-Rosenfeld Y, Yabuta N, Lapi E, Nojima H, Lu X et al. The Lats2 tumor suppressor augments p53-mediated apoptosis by promoting the nuclear proapoptotic function of ASPP1. Genes Dev 2010; 24: 2420-2429.
37. Wang G, Jiang Q, Zhang C. The role of mitotic kinases in coupling the centrosome cycle with the assembly of the mitotic spindle. J Cell Sci 2014; 127: 4111-4122.

38. Toji S, Yabuta N, Hosomi T, Nishihara S, Kobayashi T, Suzuki S et al. The centrosomal protein Lats2 is a phosphorylation target of Aurora-A kinase. Genes Cells 2004; 9: 383-397.

39. Yabuta N, Mukai S, Okada N, Aylon Y, Nojima H. The tumor suppressor Lats2 is pivotal in Aurora A and Aurora B signaling during mitosis. Cell Cycle 2011; 10: 2724-2736.

40. Zhang L, lyer J, Chowdhury A, Ji M, Xiao L, Yang S et al. KIBRA regulates aurora kinase activity and is required for precise chromosome alignment during mitosis. J Biol Chem 2012; 287: 34069-34077.

41. Humbert N, Navaratnam N, Augert A, Da Costa M, Martien S, Wang J et al. Regulation of ploidy and senescence by the AMPK-related kinase NUAK1. EMBO J 2010; 29: 376-386.

42. Xiao L, Chen Y, Ji M, Dong J. KIBRA regulates Hippo signaling activity via interactions with large tumor suppressor kinases. J Biol Chem 2011; 286: 7788-7796.

43. Huntoon CJ, Nye MD, Geng L, Peterson KL, Flatten KS, Haluska P et al. Heat shock protein 90 inhibition depletes LATS1 and LATS2, two regulators of the mammalian Hippo tumor suppressor pathway. Cancer Res 2010; 70: 8642-8650.

44. Das Thakur M, Feng Y, Jagannathan R, Seppa MJ, Skeath JB, Longmore GD. Ajuba LIM proteins are negative regulators of the Hippo signaling pathway. Curr Biol 2010; 20: 657-662.

45. Rauskolb C, Pan G, Reddy BVVG, Oh H, Irvine KD. Zyxin links fat signaling to the Hippo pathway. PLOS Biol 2011; 9: e1000624.

46. Bae SJ, Kim M, Kim S-H, Kwon YE, Lee J-H, Kim J et al. NEDD4 controls intestinal stem cell homeostasis by regulating the Hippo signalling pathway. Nat Commun 2015; 6: 6314 .

47. Salah Z, Cohen S, Itzhaki E, Aqeilan RI. NEDD4 E3 ligase inhibits the activity of the Hippo pathway by targeting LATS1 for degradation. Cell Cycle 2013; 12: 3817-3823.

48. Ho KC, Zhou Z, She Y-M, Chun A, Cyr TD, Yang X. Itch E3 ubiquitin ligase regulates large tumor suppressor 1 stability [corrected]. Proc Natl Acad Sci USA 2011; 108: 4870-4875.

49. Salah Z, Melino G, Aqeilan RI. Negative regulation of the Hippo pathway by E3 ubiquitin ligase ITCH is sufficient to promote tumorigenicity. Cancer Res 2011; 71: 2010-2020.

50. Yeung B, Ho K-C, Yang X. WWP1 E3 ligase targets LATS1 for ubiquitin-mediated degradation in breast cancer cells. PLOS ONE 2013; 8: e61027.

51. Li W, Cooper J, Zhou L, Yang C, Erdjument-Bromage H, Zagzag D et al. Merlin/NF2 lossdriven tumorigenesis linked to CRL4(DCAF1)-mediated inhibition of the Hippo pathway kinases Lats 1 and 2 in the nucleus. Cancer Cell 2014; 26: 48-60.

52. Ma B, Chen Y, Chen L, Cheng H, Mu C, Li J et al. Hypoxia regulates Hippo signalling through the SIAH2 ubiquitin E3 ligase. Nat Cell Biol 2015; 17: 95-103.

53. Nakayama K, Qi J, Ronai Z. The ubiquitin ligase Siah2 and the hypoxia response. Mol Cancer Res 2009; 7: 443-451.

54. Takahashi A, Ohtani N, Yamakoshi K, lida S, Tahara H, Nakayama K et al. Mitogenic signalling and the p16INK4a-Rb pathway cooperate to enforce irreversible cellular senescence. Nat Cell Biol 2006; 8: 1291-1297.

55. Cooke IE, Shelling AN, Le Meuth VG, Charnock ML, Ganesan TS. Allele loss on chromosome arm $6 \mathrm{q}$ and fine mapping of the region at $6 \mathrm{q} 27$ in epithelial ovarian cancer. Genes Chromosomes Cancer 1996; 15: 223-233.

56. Lee JH, Kavanagh JJ, Wildrick DM, Wharton JT, Blick M. Frequent loss of heterozygosity on chromosomes 6q, 11, and 17 in human ovarian carcinomas. Cancer Res 1990; 50: 2724-2728.

57. Mazurenko N, Attaleb M, Gritsko T, Semjonova L, Pavlova L, Sakharova O et al. High resolution mapping of chromosome 6 deletions in cervical cancer. Oncol Rep 1999; 6: 859-863.

58. Fujii H, Zhou W, Gabrielson E. Detection of frequent allelic loss of 6q23-q25.2 in microdissected human breast cancer tissues. Genes Chromosomes Cancer 1996; 16: 35-39.

59. Theile M, Seitz S, Arnold W, Jandrig B, Frege R, Schlag PM et al. A defined chromosome $6 \mathrm{q}$ fragment (at D6S310) harbors a putative tumor suppressor gene for breast cancer. Oncogene 1996; 13: 677-685.

60. Noviello C, Courjal F, Theillet C. Loss of heterozygosity on the long arm of chromosome 6 in breast cancer: possibly four regions of deletion. Clin Cancer Res 1996; 2: 1601-1606.

61. Lee EY, To H, Shew JY, Bookstein R, Scully P, Lee WH. Inactivation of the retinoblastoma susceptibility gene in human breast cancers. Science 1988; 241: 218-221.

62. Sato T, Saito H, Morita R, Koi S, Lee JH, Nakamura Y. Allelotype of human ovarian cancer. Cancer Res 1991; 51: 5118-5122.

63. Wang HP, Rogler CE. Deletions in human chromosome arms $11 p$ and $13 q$ in primary hepatocellular carcinomas. Cytogenet Cell Genet 1988; 48: 72-78.

64. Chen C-F, Yeh S-H, Chen D-S, Chen P-J, Jou Y-S. Molecular genetic evidence supporting a novel human hepatocellular carcinoma tumor suppressor locus at 13q12.11. Genes Chromosomes Cancer 2005; 44: 320-328.

65. Strazisar M, Mlakar V, Glavac D. LATS2 tumour specific mutations and down-regulation of the gene in non-small cell carcinoma. Lung Cancer 2009; 64: 257-262.

66. Ouillette P, Erba H, Kujawski L, Kaminski M, Shedden K, Malek SN. Integrated genomic profiling of chronic lymphocytic leukemia identifies subtypes of deletion 13q14. Cancer Res 2008; 68: 1012-1021.

67. Yu T, Bachman J, Lai Z-C. Mutation analysis of large tumor suppressor genes LATS1 and LATS2 supports a tumor suppressor role in human cancer. Protein Cell 2015; 6: 6-11.

68. Bonilla X, Parmentier L, King B, Bezrukov F, Kaya G, Zoete V et al. Genomic analysis identifies new drivers and progression pathways in skin basal cell carcinoma. Nat Genet 2016; 48: 398-406 
69. Ishizaki K, Fujimoto J, Kumimoto H, Nishimoto Y, Shimada Y, Shinoda M et al. Frequent polymorphic changes but rare tumor specific mutations of the LATS2 gene on 13q11-12 in esophageal squamous cell carcinoma. Int J Oncol 2002; 21: 1053-1057.

70. Esteller M. Epigenetic gene silencing in cancer: the DNA hypermethylome. Hum Mol Genet 2007; 16(Spec No 1): R50-R59.

71. Reddy VR, Annamalai T, Narayanan V, Ramanathan A. Hypermethylation of promoter region of LATS1-a CDK interacting protein in oral squamous cell carcinomas-a pilot study in India. Asian Pac J Cancer Prev 2015; 16: 1599-1603.

72. Wierzbicki PM, Adrych K, Kartanowicz D, Stanislawowski M, Kowalczyk A, Godlewski J et al. Underexpression of LATS1 TSG in colorectal cancer is associated with promoter hypermethylation. World J Gastroenterol 2013; 19: 4363-4373.

73. Chen K-H, He J, Wang D-L, Cao J-J, Li M-C, Zhao X-M et al. Methylation-associated inactivation of LATS1 and its effect on demethylation or overexpression on YAP and cell biological function in human renal cell carcinoma. Int $J$ Oncol 2014; 45: 2511-2521.

74. Yao F, Liu H, Li Z, Zhong C, Fang W. Down-regulation of LATS2 in non-small cell lung cancer promoted the growth and motility of cancer cells. Tumour Biol 2015; 36 : 2049-2057.

75. Jiménez-Velasco A, Román-Gómez J, Agirre X, Barrios M, Navarro G, Vázquez I et al. Downregulation of the large tumor suppressor 2 (LATS2/KPM) gene is associated with poor prognosis in acute lymphoblastic leukemia. Leukemia 2005; 19: 2347-2350.

76. Takahashi Y, Miyoshi Y, Takahata C, Irahara N, Taguchi T, Tamaki Y et al. Down-regulation of LATS1 and LATS2 mRNA expression by promoter hypermethylation and its association with biologically aggressive phenotype in human breast cancers. Clin Cancer Res 2005; 11 : $1380-1385$

77. Steinmann K, Sandner A, Schagdarsurengin U, Dammann RH. Frequent promoter hypermethylation of tumor-related genes in head and neck squamous cell carcinoma. Oncol Rep 2009; 22: 1519-1526.

78. Jiang Z, Li X, Hu J, Zhou W, Jiang Y, Li G et al. Promoter hypermethylation-mediated down-regulation of LATS1 and LATS2 in human astrocytoma. Neurosci Res 2006; 56: 450-458.

79. Sasaki H, Hikosaka Y, Kawano O, Yano M, Fujii Y. Hypermethylation of the large tumor suppressor genes in Japanese lung cancer. Oncol Lett 2010; 1: 303-307.

80. Ladiz MAR, Najafi M, Kordi-Tamandani DM. Contribution of LATS1 and LATS2 promoter methylation in OSCC development. J Cell Commun Signal 2017; 11: 49-55.

81. Oh J-E, Ohta T, Satomi K, Foll M, Durand G, McKay J et al. Alterations in the NF2/LATS1/LATS2/YAP pathway in schwannomas. J Neuropathol Exp Neurol 2015; 74: 952-959.

82. Malik SA, Khan MS, Dar M, Hussain MU, Shah MA, Shafi SM et al. Molecular alterations and expression dynamics of LATS1 and LATS2 genes in non-small-cell lung carcinoma. Pathol Oncol Res 2017

83. Liang R, Lin Y, Yuan C-L, Liu Z-H, Li Y-Q, Luo X-L et al. The clinical significance and biological function of large tumour suppressor 2 in hepatocellular carcinoma. Cell Prolif $2017 ; 50$

84. Wan L, Sun M, Liu G-J, Wei C-C, Zhang E-B, Kong R et al. Long noncoding RNA PVT1 promotes non-small cell lung cancer cell proliferation through epigenetically regulating LATS2 expression. Mol Cancer Ther 2016; 15: 1082-1094.

85. Huang M, Hou J, Wang Y, Xie M, Wei C, Nie F et al. Long noncoding RNA LINC00673 is activated by SP1 and exerts oncogenic properties by interacting with LSD1 and EZH2 in gastric cancer. Mol Ther 2017; 25: 1014-1026.

86. Li W, Sun M, Zang C, Ma P, He J, Zhang $M$ et al. Upregulated long non-coding RNA AGAP2-AS1 represses LATS2 and KLF2 expression through interacting with EZH2 and LSD1 in non-small-cell lung cancer cells. Cell Death Dis 2016; 7: e2225.

87. Kostic $\mathrm{C}$, Shaw $\mathrm{PH}$. Isolation and characterization of sixteen novel p53 response genes. Oncogene 2000; 19: 3978-3987.

88. Aylon Y, Oren M. The Paradox of p53: what, how, and why? Cold Spring Harb Perspect Med 2016; 6: a026328.

89. Aylon Y, Michael D, Shmueli A, Yabuta N, Nojima H, Oren M. A positive feedback loop between the p53 and Lats2 tumor suppressors prevents tetraploidization. Genes Dev 2006; 20: 2687-2700.

90. Aylon Y, Gershoni A, Rotkopf R, Biton IE, Porat Z, Koh AP et al. The LATS2 tumor suppressor inhibits SREBP and suppresses hepatic cholesterol accumulation. Genes Dev 2016; 30: 786-797

91. Aylon Y, Sarver A, Tovy A, Ainbinder E, Oren M. Lats2 is critical for the pluripotency and proper differentiation of stem cells. Cell Death Differ 2014; 21: 624-633.

92. Li W, Wang L, Katoh H, Liu R, Zheng P, Liu Y. Identification of a tumor suppressor relay between the FOXP3 and the Hippo pathways in breast and prostate cancers. Cancer Res 2011; 71: 2162-2171.

93. Du X, Shi H, Li J, Dong Y, Liang J, Ye J et al. Mst1/Mst2 regulate development and function of regulatory $T$ cells through modulation of Fox01/Foxo3 stability in autoimmune disease. $\mathrm{J}$ Immunol 2014; 192: 1525-1535.

94. Park G-S, Oh H, Kim M, Kim T, Johnson RL, Irvine KD et al. An evolutionarily conserved negative feedback mechanism in the Hippo pathway reflects functional difference between LATS1 and LATS2. Oncotarget 2016; 7: 24063-24075.

95. Moroishi T, Park HW, Qin B, Chen Q, Meng Z, Plouffe SW et al. A YAP/TAZ-induced feedback mechanism regulates Hippo pathway homeostasis. Genes Dev 2015; 29: $1271-1284$
96. Chen Q, Zhang N, Xie R, Wang W, Cai J, Choi K-S et al. Homeostatic control of Hippo signaling activity revealed by an endogenous activating mutation in YAP. Genes Dev 2015 29: $1285-1297$.

97. Sansregret L, Nepveu A. The multiple roles of CUX1: insights from mouse models and cell-based assays. Gene 2008; 412: 84-94.

98. Siam R, Harada R, Cadieux C, Battat R, Vadnais C, Nepveu A. Transcriptional activation of the Lats1 tumor suppressor gene in tumors of CUX1 transgenic mice. Mol Cancer 2009; 8 : 60.

99. Zhang Y, Hu C-F, Chen J, Yan L-X, Zeng Y-X, Shao J-Y. LATS2 is de-methylated and overexpressed in nasopharyngeal carcinoma and predicts poor prognosis. BMC Cancer 2010; 10: 538.

100. Zhang K, Rodriguez-Aznar E, Yabuta N, Owen RJ, Mingot JM, Nojima H et al. Lats2 kinase potentiates Snail 1 activity by promoting nuclear retention upon phosphorylation. EMBO J 2012; 31: 29-43.

101. Furth N, Bossel Ben-Moshe N, Pozniak Y, Porat Z, Geiger T, Domany E et al Down-regulation of LATS kinases alters p53 to promote cell migration. Genes Dev2015; 29: 2325-2330.

102. Zhu R, Lacovino M, Mahen E, Kyba M, Matin A. Transcripts that associate with the RNA binding protein, DEAD-END (DND1), in embryonic stem (ES) cells. BMC Mol Biol 2011; 12:

103. Wu Q, Ma Q, Shehadeh LA, Wilson A, Xia L, Yu H et al. Expression of the Argonaute protein PiwiL2 and piRNAs in adult mouse mesenchymal stem cells. Biochem Biophys Res Commun 2010; 396: 915-920.

104. Kedde M, Strasser MJ, Boldajipour B, Oude Vrielink JAF, Slanchev K, le Sage C et al. RNA-binding protein Dnd1 inhibits microRNA access to target mRNA. Cell 2007; 131: 1273-1286.

105. Ali S, Karki N, Bhattacharya C, Zhu R, MacDuff DA, Stenglein MD et al. APOBEC3 inhibits DEAD-END function to regulate microRNA activity. BMC Mol Biol 2013; 14: 16.

106. Lee HH, Vo M-T, Kim HJ, Lee UH, Kim CW, Kim HK et al. Stability of the LATS2 tumor suppressor gene is regulated by tristetraprolin. J Biol Chem 2010; 285 17329-17337.

107. Hua K, Jin J, Zhao J, Song J, Song H, Li D et al. miR-135b, upregulated in breast cancer, promotes cell growth and disrupts the cell cycle by regulating LATS2. Int J Oncol 2016; 48 : 1997-2006.

108. Lin C-W, Chang Y-L, Chang Y-C, Lin J-C, Chen C-C, Pan S-H et al. MicroRNA-135b promotes lung cancer metastasis by regulating multiple targets in the Hippo pathway and LZTS1. Nat Commun 2013; 4: 1877.

109. Liu X, Sempere LF, Ouyang H, Memoli VA, Andrew AS, Luo Y et al. MicroRNA-31 functions as an oncogenic microRNA in mouse and human lung cancer cells by repressing specific tumor suppressors. J Clin Invest 2010; 120: 1298-1309.

110. Mitamura $\mathrm{T}$, Watari $\mathrm{H}$, Wang L, Kanno $\mathrm{H}$, Kitagawa M, Hassan MK et al. microRNA 31 functions as an endometrial cancer oncogene by suppressing Hippo tumor suppressor pathway. Mol Cancer 2014; 13: 97.

111. Voorhoeve PM, le Sage C, Schrier M, Gillis AJM, Stoop H, Nagel R et al. A genetic screen implicates miRNA-372 and miRNA-373 as oncogenes in testicular germ cell tumors. Cell 2006; 124: 1169-1181.

112. Cho WJ, Shin JM, Kim JS, Lee MR, Hong KS, Lee J-H et al. miR-372 regulates cell cycle and apoptosis of ags human gastric cancer cell line through direct regulation of LATS2. Mol Cells 2009; 28: 521-527.

113. Lee K-H, Goan Y-G, Hsiao M, Lee C-H, Jian S-H, Lin J-T et al. MicroRNA-373 (miR-373) post-transcriptionally regulates large tumor suppressor, homolog 2 (LATS2) and stimulates proliferation in human esophageal cancer. Exp Cell Res 2009; 315 : 2529-2538.

114. Luo Z-L, Luo H-J, Fang C, Cheng L, Huang Z, Dai R et al. Negative correlation of ITCH E3 ubiquitin ligase and miRNA-106b dictates metastatic progression in pancreatic cancer. Oncotarget 2016; 7: 1477-1485.

115. Deng J, Lei W, Xiang X, Zhang L, Lei J, Gong Y et al. Cullin 4A (CUL4A), a direct target of $m i R-9$ and miR-137, promotes gastric cancer proliferation and invasion by regulating the Hippo signaling pathway. Oncotarget 2016; 7: 10037-10050.

116. Higashi $\mathrm{T}$, Hayashi $\mathrm{H}$, Ishimoto $\mathrm{T}$, Takeyama $\mathrm{H}$, Kaida $\mathrm{T}$, Arima $\mathrm{K}$ et al. miR-9-3p plays a tumour-suppressor role by targeting TAZ (WWTR1) in hepatocellular carcinoma cells. Br J Cancer 2015; 113: 252-258.

117. Kwon Y, Vinayagam A, Sun X, Dephoure N, Gygi SP, Hong P et al. The Hippo signaling pathway interactome. Science 2013; 342: 737-740.

118. Wang W, Xiao Z-D, Li X, Aziz KE, Gan B, Johnson RL et al. AMPK modulates Hippo pathway activity to regulate energy homeostasis. Nat Cell Biol 2015; 17: 490-499.

119. Mo J-S, Meng Z, Kim YC, Park HW, Hansen CG, Kim S et al. Cellular energy stress induces AMPK-mediated regulation of YAP and the Hippo pathway. Nat Cell Biol 2015; 17: 500-510.

120. Pefani D-E, Latusek R, Pires I, Grawenda AM, Yee KS, Hamilton G et al. RASSF1A-LATS1 signalling stabilizes replication forks by restricting CDK2-mediated phosphorylation of BRCA2. Nat Cell Biol 2014; 16: 962-971 1.

121. Matsuoka S, Ballif BA, Smogorzewska A, McDonald ER, Hurov KE, Luo J et al. ATM and ATR substrate analysis reveals extensive protein networks responsive to DNA damage. Science 2007; 316: 1160-1166.

122. Wang W, Li X, Huang J, Feng L, Dolinta KG, Chen J. Defining the protein-protein interaction network of the human Hippo pathway. Mol Cell Proteomics 2014; 13: 119-131. 
123. Couzens AL, Knight JDR, Kean MJ, Teo G, Weiss A, Dunham WH et al. Protein interaction network of the mammalian Hippo pathway reveals mechanisms of kinase-phosphatase interactions. Sci Signal 2013; 6: rs15.

124. Hirate Y, Hirahara S, Inoue K-I, Suzuki A, Alarcon VB, Akimoto K et al. Polarity-dependent distribution of angiomotin localizes Hippo signaling in preimplantation embryos. Curr Biol 2013; 23: 1181-1194.

125. Yang X, Yu K, Hao Y, Li D, Stewart R, Insogna KL et al. LATS1 tumour suppressor affects cytokinesis by inhibiting LIMK1. Nat Cell Biol 2004; 6: 609-617.

126. Lee J-H, Kim T-S, Yang T-H, Koo B-K, Oh S-P, Lee K-P et al. A crucial role of WW45 in developing epithelial tissues in the mouse. EMBO J 2008; 27: 1231-1242.

127. Zhang $P$, Wang S, Wang S, Qiao J, Zhang L, Zhang Z et al. Dual function of partitioning defective 3 in the regulation of YAP phosphorylation and activation. Cell Discov 2016; 2 16021.

128. Britschgi A, Duss S, Kim S, Couto JP, Brinkhaus H, Koren S et al. The Hippo kinases LATS1 and 2 control human breast cell fate via crosstalk with ER $\alpha$. Nature 2017; 541: $541-545$.

129. Torigata K, Daisuke O, Mukai S, Hatanaka A, Ohka F, Motooka D et al. LATS2 positively regulates polycomb repressive complex 2. PLOS ONE 2016; 11: e0158562.

130. Li J, Chen X, Ding X, Cheng Y, Zhao B, Lai Z-C et al. LATS2 suppresses oncogenic Wnt signaling by disrupting $\beta$-catenin/BCL9 interaction. Cell Rep 2013; 5: 1650-1663.

131. Heallen T, Zhang M, Wang J, Bonilla-Claudio M, Klysik E, Johnson RL et al. Hippo pathway inhibits Wnt signaling to restrain cardiomyocyte proliferation and heart size. Science 2011; 332: 458-461.

132. Powzaniuk M, McElwee-Witmer S, Vogel RL, Hayami T, Rutledge SJ, Chen F et al. The LATS2/KPM tumor suppressor is a negative regulator of the androgen receptor. Mol Endocrinol 2004; 18: 2011-2023.

133. Lit LC, Scott S, Zhang H, Stebbing J, Photiou A, Giamas G. LATS2 is a modulator of estrogen receptor alpha. Anticancer Res 2013; 33: 53-63.

134. Visser S, Yang X. LATS tumor suppressor: a new governor of cellular homeostasis. Cell Cycle 2010; 9: 3892-3903.

135. Tschöp K, Conery AR, Litovchick L, Decaprio JA, Settleman J, Harlow E et al. A kinase shRNA screen links LATS2 and the pRB tumor suppressor. Genes Dev 2011; 25: 814-830.

136. Mukai S, Yabuta N, Yoshida K, Okamoto A, Miura D, Furuta Y et al. Lats1 suppresses centrosome overduplication by modulating the stability of Cdc25B. Sci Rep 2015; $\mathbf{5}: 16173$.

137. Masuda K, Chiyoda T, Sugiyama N, Segura-Cabrera A, Kabe Y, Ueki A et al. LATS1 and LATS2 phosphorylate CDC26 to modulate assembly of the tetratricopeptide repeat subcomplex of APC/C. PLOS ONE 2015; 10: e0118662.

138. Kamikubo Y, Takaori-Kondo A, Uchiyama T, Hori T. Inhibition of cell growth by conditional expression of kpm, a human homologue of Drosophila warts/lats tumor suppressor. J Biol Chem 2003; 278: 17609-17614.

139. Yang X, Li DM, Chen W, Xu T. Human homologue of Drosophila lats, LATS1, negatively regulate growth by inducing $\mathrm{G}(2) / \mathrm{M}$ arrest or apoptosis. Oncogene $2001 ; 20$ : 6516-6523.

140. Xia H, Qi H, Li Y, Pei J, Barton J, Blackstad M et al. LATS1 tumor suppressor regulates G2 $M$ transition and apoptosis. Oncogene 2002; 21: 1233-1241.

141. Zhao B, Li L, Wang L, Wang C-Y, Yu J, Guan K-L. Cell detachment activates the Hippo pathway via cytoskeleton reorganization to induce anoikis. Genes Dev 2012; 26: 54-68.

142. Ganem NJ, Cornils H, Chiu S-Y, O'Rourke KP, Arnaud J, Yimlamai D et al. Cytokinesis failure triggers Hippo tumor suppressor pathway activation. Cell 2014; 158: 833-848.

143. Vigneron AM, Vousden $\mathrm{KH}$. An indirect role for ASPP1 in limiting p53-dependent p21 expression and cellular senescence. EMBO J 2012; 31: 471-480.

144. lida S-I, Hirota T, Morisaki T, Marumoto T, Hara T, Kuninaka S et al. Tumor suppressor WARTS ensures genomic integrity by regulating both mitotic progression and G1 tetraploidy checkpoint function. Oncogene 2004; 23: 5266-5274.

145. Haupt $Y$, Maya R, Kazaz A, Oren M. Mdm2 promotes the rapid degradation of $p 53$. Nature 1997; 387: 296-299.

146. Matallanas D, Romano D, Al-Mulla F, O'Neill E, Al-Ali W, Crespo P et al. Mutant K-Ras activation of the proapoptotic MST2 pathway is antagonized by wild-type K-Ras. Mol Cell 2011; 44: 893-906.

147. Vigneron AM, Ludwig RL, Vousden KH. Cytoplasmic ASPP1 inhibits apoptosis through the control of YAP. Genes Dev 2010; 24: 2430-2439.

148. Matallanas D, Romano D, Yee K, Meiss K, Kucerova L, Piazzolla D et al. RASSF1A elicits apoptosis through an MST2 pathway directing proapoptotic transcription by the p73 tumor suppressor protein. Mol Cell 2007; 27: 962-975.

149. Kuninaka S, Nomura M, Hirota T, lida S-I, Hara T, Honda S et al. The tumor suppressor WARTS activates the Omi/HtrA2-dependent pathway of cell death. Oncogene 2005; 24: 5287-5298.

150. Hegde R, Srinivasula SM, Zhang Z, Wassell R, Mukattash R, Cilenti L et al. Identification of Omi/HtrA2 as a mitochondrial apoptotic serine protease that disrupts inhibitor of apoptosis protein-caspase interaction. J Biol Chem 2002; 277: 432-438.

151. Romano D, Nguyen LK, Matallanas D, Halasz M, Doherty C, Kholodenko BN et al. Protein interaction switches coordinate Raf-1 and MST2/Hippo signalling. Nat Cell Biol 2014; 16: 673-684.

152. Ke H, Pei J, Ni Z, Xia H, Qi H, Woods T et al. Putative tumor suppressor Lats2 induces apoptosis through downregulation of Bcl-2 and Bcl-x(L). Exp Cell Res 2004; 298: 329-338.

153. Suzuki H, Yabuta N, Okada N, Torigata K, Aylon Y, Oren M et al. Lats2 phosphorylates p21/ CDKN1A after UV irradiation and regulates apoptosis. J Cell Sci 2013; 126: 4358-4368.
154. Strano S, Monti O, Pediconi N, Baccarini A, Fontemaggi G, Lapi E et al. The transcriptional coactivator Yes-associated protein drives p73 gene-target specificity in response to DNA damage. Mol Cell 2005; 18: 447-459.

155. Basu S, Totty NF, Irwin MS, Sudol M, Downward J. Akt phosphorylates the Yes-associated protein, YAP, to induce interaction with 14-3-3 and attenuation of p73-mediated apoptosis. Mol Cell 2003; 11: 11-23.

156. Kawahara M, Hori T, Chonabayashi K, Oka T, Sudol M, Uchiyama T. Kpm/Lats2 is linked to chemosensitivity of leukemic cells through the stabilization of p73. Blood 2008; 112: 3856-3866.

157. Oka T, Mazack V, Sudol M. Mst2 and Lats kinases regulate apoptotic function of Yes kinase-associated protein (YAP). J Biol Chem 2008; 283: 27534-27546.

158. Reuven N, Adler J, Meltser V, Shaul Y. The Hippo pathway kinase Lats2 prevents DNA damage-induced apoptosis through inhibition of the tyrosine kinase c-Abl. Cell Death Differ 2013; 20: 1330-1340.

159. Levy D, Adamovich Y, Reuven N, Shaul Y. Yap1 phosphorylation by c-Abl is a critical step in selective activation of proapoptotic genes in response to DNA damage. Mol Cell 2008; 29: 350-361.

160. Agami R, Blandino G, Oren M, Shaul Y. Interaction of c-Abl and p73alpha and their collaboration to induce apoptosis. Nature 1999; 399: 809-813.

161. Strano S, Munarriz E, Rossi M, Castagnoli L, Shaul Y, Sacchi A et al. Physical interaction with Yes-associated protein enhances p73 transcriptional activity. J Biol Chem 2001; 276: 15164-15173.

162. Sirvent A, Benistant $C$, Roche S. Cytoplasmic signalling by the c-Abl tyrosine kinase in normal and cancer cells. Biol Cell 2008; 100: 617-631.

163. Dong J, Feldmann G, Huang J, Wu S, Zhang N, Comerford SA et al. Elucidation of a universal size-control mechanism in Drosophila and mammals. Cell 2007; 130: 1120-1133.

164. Visser-Grieve S, Zhou Z, She Y-M, Huang H, Cyr TD, Xu T et al. LATS1 tumor suppressor is a novel actin-binding protein and negative regulator of actin polymerization. Cell Res 2011; 21: $1513-1516$.

165. Fang X, Adler PN. Regulation of cell shape, wing hair initiation and the actin cytoskeleton by Trc/Fry and Wts/Mats complexes. Dev Biol 2010; 341: 360-374.

166. Moroishi T, Hansen CG, Guan K-L. The emerging roles of YAP and TAZ in cancer. Nat Rev Cancer 2015; 15: 73-79.

167. Dupont S, Morsut L, Aragona M, Enzo E, Giulitti S, Cordenonsi M et al. Role of YAP/TAZ in mechanotransduction. Nature 2011; 474: 179-183.

168. Varelas X, Samavarchi-Tehrani P, Narimatsu M, Weiss A, Cockburn K, Larsen BG et al. The Crumbs complex couples cell density sensing to Hippo-dependent control of the TGF- $\beta$ SMAD pathway. Dev Cell 2010; 19: 831-844.

169. Nishioka N, Inoue K, Adachi K, Kiyonari H, Ota M, Ralston A et al. The Hippo signaling pathway components Lats and Yap pattern Tead4 activity to distinguish mouse trophectoderm from inner cell mass. Dev Cell 2009; 16: 398-410.

170. Lorthongpanich C, Messerschmidt DM, Chan SW, Hong W, Knowles BB, Solter D. Temporal reduction of LATS kinases in the early preimplantation embryo prevents ICM lineage differentiation. Genes Dev 2013; 27: 1441-1446.

171. Loh Y-H, Wu Q, Chew J-L, Vega VB, Zhang W, Chen X et al. The Oct4 and Nanog transcription network regulates pluripotency in mouse embryonic stem cells. Nat Genet 2006; 38: 431-440.

172. Wang Y, Baskerville S, Shenoy A, Babiarz JE, Baehner L, Blelloch R. Embryonic stem cell-specific microRNAs regulate the G1-S transition and promote rapid proliferation. Nat Genet 2008; 40: 1478-1483.

173. Qin H, Blaschke K, Wei G, Ohi Y, Blouin L, Qi Z et al. Transcriptional analysis of pluripotency reveals the Hippo pathway as a barrier to reprogramming. Hum Mol Genet 2012; 21: 2054-2067.

174. An Y, Kang Q, Zhao Y, Hu X, Li N. Lats2 modulates adipocyte proliferation and differentiation via Hippo signaling. PLOS ONE 2013; 8: e72042.

175. Liu Q, Gu X, Zhao Y, Zhang J, Zhao Y, Meng Q et al. Pig large tumor suppressor 2 (Lats2), a novel gene that may regulate the fat reduction in adipocyte. BMB Rep 2010; 43: 97-102.

176. Yimlamai D, Christodoulou C, Galli GG, Yanger K, Pepe-Mooney B, Gurung B et al. Hippo pathway activity influences liver cell fate. Cell 2014; 157: 1324-1338.

177. Yi J, Lu L, Yanger K, Wang W, Sohn BH, Stanger BZ et al. Large tumor suppressor homologs 1 and 2 regulate mouse liver progenitor cell proliferation and maturation through antagonism of the coactivators YAP and TAZ. Hepatology 2016; 64: 1757-1772.

178. Lee D-H, Park JO, Kim T-S, Kim S-K, Kim T-H, Kim M-C et al. LATS-YAP/TAZ controls lineage specification by regulating TGF $\beta$ signaling and $H n f 4 \alpha$ expression during liver development. Nat Commun 2016; 7: 11961

179. Reginensi A, Enderle L, Gregorieff A, Johnson RL, Wrana JL, McNeill H. A critical role for NF2 and the Hippo pathway in branching morphogenesis. Nat Commun 2016; 7: 12309

180. Heallen T, Morikawa Y, Leach J, Tao G, Willerson JT, Johnson RL et al. Hippo signaling impedes adult heart regeneration. Development 2013; 140: 4683-4690.

181. Matsui Y, Nakano N, Shao D, Gao S, Luo W, Hong C et al. Lats2 is a negative regulator of myocyte size in the heart. Circ Res 2008; 103: 1309-1318.

182. Singh A, Ramesh S, Cibi DM, Yun LS, Li J, Li L et al. Hippo signaling mediators yap and taz are required in the epicardium for coronary vasculature development. Cell Rep 2016; 15 : 1384-1393.

183. Uhlén M, Fagerberg L, Hallström BM, Lindskog C, Oksvold $P$, Mardinoglu A et al. Proteomics. tissue-based map of the human proteome. Science 2015; 347: 1260419. 
184. Gu Z, Steinmetz LM, Gu X, Scharfe C, Davis RW, Li W-H. Role of duplicate genes in genetic robustness against null mutations. Nature 2003; 421: 63-66.

185. Li J, Musso G, Zhang Z. Preferential regulation of duplicated genes by microRNAs in mammals. Genome Biol 2008; 9: R132.

186. Ohno S. Evolution by Gene Duplication. Springer Berlin Heidelberg: Berlin, Germany, 1970.

187. Li W-H, Yang J, Gu X. Expression divergence between duplicate genes. Trends Genet 2005; 21: 602-607.

188. Chen C-Y, Chen S-T, Juan H-F, Huang H-C. Lengthening of 3'UTR increases with morphological complexity in animal evolution. Bioinformatics 2012; 28: 3178-3181.

189. Hausser J, Zavolan M. Identification and consequences of miRNA-target interactionsbeyond repression of gene expression. Nat Rev Genet 2014; 15: 599-612.

190. Subramanyam D, Lamouille S, Judson RL, Liu JY, Bucay N, Derynck R et al. Multiple targets of miR-302 and miR-372 promote reprogramming of human fibroblasts to induced pluripotent stem cells. Nat Biotechnol 2011; 29: 443-448.

191. Carmena M, Ruchaud S, Earnshaw WC. Making the Auroras glow: regulation of Aurora A and B kinase function by interacting proteins. Curr Opin Cell Biol 2009; 21 : 796-805

192. Hao Y, Chun A, Cheung K, Rashidi B, Yang X. Tumor suppressor LATS1 is a negative regulator of oncogene YAP. J Biol Chem 2008; 283: 5496-5509.

193. Chou C-H, Chang N-W, Shrestha S, Hsu S-D, Lin Y-L, Lee W-H et al. miRTarBase 2016 updates to the experimentally validated miRNA-target interactions database. Nucleic Acids Res 2016; 44: D239-D247.

194. Beausoleil SA, Jedrychowski M, Schwartz D, Elias JE, Villén J, Li J et al. Large-scale characterization of HeLa cell nuclear phosphoproteins. Proc Natl Acad Sci USA 2004; 101: 12130-12135.

195. Emery A, Sorrell DA, Lawrence S, Easthope E, Stockdale M, Jones DO et al. A novel cell-based, high-content assay for phosphorylation of Lats2 by Aurora A. J Biomol Screen 2011; 16: 925-931.

196. Van Hoof D, Muñoz J, Braam SR, Pinkse MWH, Linding R, Heck AJR et al. Phosphorylation dynamics during early differentiation of human embryonic stem cells. Cell Stem Cell 2009; 5: 214-226.

197. Zhou H, Di Palma S, Preisinger C, Peng M, Polat AN, Heck AJR et al. Toward a comprehensive characterization of a human cancer cell phosphoproteome. J Proteome Res 2013; 12: 260-271.

198. Dephoure N, Zhou C, Villén J, Beausoleil SA, Bakalarski CE, Elledge SJ et al. A quantitative atlas of mitotic phosphorylation. Proc Natl Acad Sci USA 2008; 105 : 10762-10767.

199. Daub H, Olsen JV, Bairlein M, Gnad F, Oppermann FS, Körner R et al. Kinase-selective enrichment enables quantitative phosphoproteomics of the kinome across the cell cycle. Mol Cell 2008; 31: 438-448.

200. Pan C, Olsen JV, Daub H, Mann M. Global effects of kinase inhibitors on signaling networks revealed by quantitative phosphoproteomics. Mol Cell Proteomics 2009; 8: 2796-2808.

201. Morisaki T, Hirota T, lida S, Marumoto T, Hara T, Nishiyama $Y$ et al. WARTS tumor suppressor is phosphorylated by $\mathrm{Cdc} /$ cyclin $\mathrm{B}$ at spindle poles during mitosis. FEBS Lett 2002; 529: 319-324.
202. Ni L, Zheng $Y$, Hara M, Pan D, Luo X. Structural basis for Mob1-dependent activation of the core Mst-Lats kinase cascade in Hippo signaling. Genes Dev 2015; 29 : 1416-1431.

203. Liu G, Yu F-X, Kim YC, Meng Z, Naipauer J, Looney DJ et al. Kaposi sarcoma-associated herpesvirus promotes tumorigenesis by modulating the Hippo pathway. Oncogene 2015 34: 3536-3546.

204. Mohseni M, Sun J, Lau A, Curtis S, Goldsmith J, Fox VL et al. A genetic screen identifies an LKB1-MARK signalling axis controlling the Hippo-YAP pathway. Nat Cell Biol 2014; 16 $108-117$.

205. Hafner M, Landthaler M, Burger L, Khorshid M, Hausser J, Berninger P et al. Transcriptome-wide identification of RNA-binding protein and microRNA target sites by PAR-CLIP. Cell 2010; 141: 129-141.

206. Zheng Y-B, Xiao K, Xiao G-C, Tong S-L, Ding Y, Wang Q-S et al. MicroRNA-103 promotes tumor growth and metastasis in colorectal cancer by directly targeting LATS2. Oncol Lett 2016; 12: 2194-2200.

207. Whisnant AW, Bogerd HP, Flores O, Ho P, Powers JG, Sharova N et al. In-depth analysis of the interaction of HIV-1 with cellular microRNA biogenesis and effector mechanisms. MBio 2013; 4: e000193.

208. Karginov FV, Hannon GJ. Remodeling of Ago2-mRNA interactions upon cellular stress reflects miRNA complementarity and correlates with altered translation rates. Genes Dev 2013; 27: 1624-1632.

209. Zhang M, Wang X, Li W, Cui Y. miR-107 and miR-25 simultaneously target LATS2 and regulate proliferation and invasion of gastric adenocarcinoma (GAC) cells. Biochem Biophys Res Commun 2015; 460: 806-812.

210. Tavazoie SF, Alarcón C, Oskarsson T, Padua D, Wang Q, Bos PD et al. Endogenous human microRNAs that suppress breast cancer metastasis. Nature 2008; 451: 147-152.

211. Ragusa M, Statello L, Maugeri M, Majorana A, Barbagallo D, Salito $L$ et al. Specific alterations of the microRNA transcriptome and global network structure in colorectal cancer after treatment with MAPK/ERK inhibitors. J Mol Med 2012; 90: 1421-1438.

212. Gabriely G, Wurdinger T, Kesari S, Esau CC, Burchard J, Linsley PS et al. MicroRNA 21 promotes glioma invasion by targeting matrix metalloproteinase regulators. Mol Cell Biol 2008; 28: 5369-5380.

213. Jiang L, Li W, Wu M, Cao S. Ciculating miRNA-21 as a biomarker predicts polycystic ovary syndrome (PCOS) in patients. Clin Lab 2015; 61: 1009-1015.

214. Fang L, Du WW, Yang W, Rutnam ZJ, Peng C, Li $H$ et al. MiR-93 enhances angiogenesis and metastasis by targeting LATS2. Cell Cycle 2012; 11: 4352-4365.

215. Kishore S, Jaskiewicz L, Burger L, Hausser J, Khorshid M, Zavolan M. A quantitative analysis of CLIP methods for identifying binding sites of RNA-binding proteins. Nat Methods 2011; 8: 559-564

216. Belair C, Baud J, Chabas S, Sharma CM, Vogel J, Staedel C et al. Helicobacter pylori interferes with an embryonic stem cell micro RNA cluster to block cell cycle progression. Silence 2011; 2 : 7

217. Xia Y, Gao Y. MicroRNA-181b promotes ovarian cancer cell growth and invasion by targeting LATS2. Biochem Biophys Res Commun 2014; 447: 446-451. 